\title{
MICRO-OPPORTUNISTIC SCHEDULING: THE MICRO-BOSS FACTORY SCHEDULER
}

\author{
Norman Sadeh
}

CMU-RI-TR-94-04

\begin{abstract}
The Robotics Institute Carnegie Mellon University

Pittsburgh, Pennsylvania 15213

To appear in "Intelligent Scheduling" book edited by M. Zweben and M. Fox, Morgan Kaufmann, 1994
\end{abstract}

Copyright $@ 1994$ Sadeh

This research was supported, in part, by the Defense Advanced Research Projects Agency under contract \#F30602-91-F-0016 and in part by grants from McDonnell Aircraft Company and Digital Equipment Corporation. 


\begin{abstract}
A major challenge for research in production management is to develop new finite-capacity scheduling techniques and tools that (1) can account more precisely for actual production management constraints and objectives, (2) are better suited for handling production contingencies, and (3) allow the user to interactively manipulate the production schedule to reflect idiosyncratic constraints and preferences not easily amenable to representation in the computer model. This paper describes Micro-Boss, a decision-support system for factory scheduling currently under development at Carnegie Mellon University. Micro-Boss aims at generating and maintaining high-quality realistic production schedules by combining powerful predictive, reactive, and interactive scheduling capabilities. Specifically, the system relies on new micro-opportunistic search heuristics that enable it to constantly revise its scheduling strategy during the construction or repair of a schedule. These search heuristics are shown to be more effective than less flexible scheduling techniques proposed in the Operations Research and Artificial Intelligence literature.
\end{abstract}




\section{Table of Contents}

1. Introduction

1.1. The Production Scheduling Problem

1.2. A Micro-Opportunistic Approach to Production Scheduling

1.3. Paper Ontline

2. A Micro-opportunistic Search Procedure

2.1. A Deterministic Scheduling Model

2.2. Overview of the Search Procedure

2.3. Look-Ahead Analysis in Micro-Boss

2.3.1. Optimixing Critical Conflicts First

2.3.2. Step 1: Reservation Optimization within a Job

2.3.3. Step 2: Building Demand Profiles to Identify Critical Resource/Time Intervals

2.4. Operation Selection

2.5. Reservation Selection

3. A Small Example

4. Reactive and Interactive Scheduling in Micro-Boss

4.1. Reactive Scheduling and Control Issues

4.2. Interactive Scheduling with Micro-Boss

5. Performance Evaluation

5.1. Comparison Against Combinations of Priority Dispatch Rules and Release Policies.

5.2. Comparison Against Coarser Opportunistic Scheduling Procedures

5.3. Evaluating the Impact of Using Biased Demand Profilles

6. Concluding Remarks 


\section{List of Figures}

Figure 1: A simple job shop problem with four jobs. Each node is labeled by the operation that it represents, its duration, and the resource that it requires.

Figure 2: An example of an unscheduled operation that absolutely needs a resource/time interval.

Figure 3: Start time distribution $\sigma_{2}^{2}(\tau)$ for operation $O_{2}^{2}$ in the initial search state for the problem defined in Figure 1.

Figure 4: Building $R_{2}$ 's aggregate demand profile in the initial search state.

Figure 5: Aggregate demands in the initial search state for each of the five resources.

Figure 6: Operation selection in the initial search state.

Flgure 7: An edited trace

Figure 8: Gantt chart of the final schedule produced by Micro-Boss.

Figure 9: The Mlcro-Boss user interface allows for interactive manipulation of schedules. By interleaving both manual and automatic scheduling decisjons, saving and comparing alternative schedules, the user can easily assess different trade-offs and locally impose ad hoc constraints or preferences that are not easily amenable to representation in the computer model.

Figure 10: Comparison of Micro-Boss and the best of 39 combinations of priority dispatch rules and release policies under 8 different scheduling conditions (10 problems were generated under each condition).

Figure 11: Comparison of Micro-Boss and two coarser opportunistic schedulers.

Figure 12: Comparison of the cost of the schedules produced by Micro-Boss and a variation of the system that used unbiased demand profiles. 


\section{List of Tables}

Table 1: Earliest acceptable release dates, due dates, latest acceptable completion dates and 9 marginal costs.

Table 2: Characteristics of the eight problem sets. 


\section{Introduction}

In a global market economy, the need for cost-efficient production management techniques is becoming more critical every day. In contrast with this need, current production management practice is too often characterized by low levels of due date satisfaction, high levels of inventory and, more generally, a state of chaos in which the computer systems that are used to provide managerial guidance do not accurately reflect the current state of affairs, because they rely on oversimplified and rigid models of the production environment. A major challenge for research in this area is to develop new production management techniques and tools that (1) can account more precisely for actual production management constraints and objectives, (2) are better suited for handling production contingencies, and (3) allow the user to interactively manipulate the production schedule to reflect idiosyncratic constraints and preferences not easily amenable to representation in the computer model. This paper describes Micro-Boss, a decision-support system for factory scheduling currently under development at Carnegie Mellon University. Micro-Boss aims at generating and maintaining high-quality realistic production schedules by combining powerful predictive, reactive, and interactive scheduling capabilities. Specifically, the system relies on new micro-opportunistic search heuristics that enable it to constantly revise its scheduling strategy during the construction or repair of a schedule. These search heuristics are shown to be more effective than less flexible scheduling techniques proposed in the Operations Research and Artificial Intelligence literature.

\subsection{The Production Scheduling Problem}

Production scheduling requires allocating resources (e.g., machines, tools, human operators) over time to a set of jobs while attending to a variety of constraints and objectives.

Typical constraints include

- functional constraints limiting the types of operations that a specific resource can perform

- capacity constraints restricting the number of jobs a resource can process at any given time

- availability constraints specifying when each resource is available (e.g., number of shifts available on a group of machines)

- precedence constraints existing between operations in a job, as specified in the job's process routing

- processing time constraints specifying how long it usually takes to perform each operation

- setup constraints requiring that each machine be in the proper configuration before 
performing a particular task (e.g., proper sets of fixtures and tools)

- time-bound constraints specifying for each job an earliest acceptable release date before which the job cannot start (e.g., because its raw materials cannot arrive earlier) and a due date by which ideally it should be delivered to a customer

Some of these constraints must be satisfied for a schedule to be valid (so-called non-relaxable or hard constraints). For instance, milling operations can only be performed on milling machines. Other groups of constraints are not always satisfiable and might need to be relaxed (so-called relaxable or soft constraints). For instance, due date constraints often need to be relaxed for a couple of jobs because of the limited capacity of the production facility. Availability constraints are another example of constraints that can be relaxed, by either working overtime or adding extra shifts. A good schedule is one that satisfies all hard constraints while selectively relaxing soft constraints to maximize performance along one or several metrics.

Two factors that critically influence the quality of a schedule are due date satisfaction and inventory levels. Missing a customer due date can result in tardiness penalties, loss of customer orders, delayed revenue receipts, etc. Inventory costs include interests on the costs of raw materials, direct inventory holding costs, interests on processing costs, etc. One often distinguishes between in-process inventory costs (also referred to as work-in-process inventory costs) and finished-goods inventory costs. Work-In-Process (WIP) inventory costs account for inventory costs resulting from orders that have not yet been completed, and finished-goods inventory costs result from completed orders that have not yet been shipped to customers.

Manufacturing contingencies such as machine breakdowns, late arrivals of raw materials, and variations in operation durations and yields further complicate production scheduling. In the face of contingencies, schedules need to be updated to reflect the new state of affairs. The sheer size of most factory scheduling problems precludes the generation of new schedules from scratch each time an unanticipated event occurs. In fact, most contingencies do not warrant such extreme actions and are best handled by repairing a portion of the existing schedule [3].

As schedules are optimized at a more detailed level, they can also become more sensitive to disruptions and require more frequent repairs. In general, there is a limit to the amount and detail of information that one can reasonably expect to represent in a computer model. For instance, a worker's preference for performing more demanding tasks in the morning might not be worth storing in the computer model and, instead, might be best accounted for by allowing the end-user to interactively manipulate the schedule.

Even under idealized conditions such as simplified objectives (e.g., minimizing total tardiness or maximizing throughput) and deterministic assumptions, scheduling has been shown to be an NP-hard problem $[12,14,11]$. Uncertainty further adds to the difficulty of the problem, and makes it even more impractical to look for optimal solutions. Instead, practical approaches to production scheduling are heuristic in nature. The next subsection briefly reviews earlier 
approaches to production scheduling; identifies some of their shortcomings; and introduces a new search paradigm, called micro-opportunistic search, that shows promise for addressing some of these shortcomings.

\subsection{A Micro-Opportunistic Approach to Production Scheduling}

To this date, the most widely used computer-based approach to production scheduling remains by far the Material Requirements Planning (MRP) or Manufacturing Resource Planning (MRPII) approach developed in the $1970 \mathrm{~s}[22,41,42]$. In this approach, demand for end-products as specified in a Master Production Schedule is exploded into time-phased requirements for component items (subassemblies, parts, raw materials, etc.) required for the production of these end-products ${ }^{1}$. Because their time-phasing logic relies on standard operation leadtimes that do not account for the actual load of the production facility, MRP systems often fail to produce realistic schedules. They sometimes overload the facility, thereby causing orders to be delivered late. In an attempt to alleviate this problem, MRP systems often pad the schedule by inserting generous "safety" leadtimes. These safety leadtimes tend to be rather arbitrary and produce unnecessarily large amounts of inventory. In fact, because they are often unrealistic and are not meant to be updated in real-time ${ }^{2}$, MRP schedules are not directly used to schedule production but rather to assign priorities to jobs $[27,40]$. These priorities in turn determine the order in which jobs are actually processed at each work center.

Shortcomings of the traditional MRP approach reflect limitations of computing technologies available in the 1970s. In the 1980 s with the advent of more powerful computers, several more sophisticated techniques emerged [13,9,23, 1, 24, 21]. The first and by far most publicized of these techniques is the one developed by Goldratt and his colleagues within the context of the OPT factory scheduling system $[13,15,10]$. OPT demonstrated the benefits of building detailed production schedules that account for the actual load of the plant and the finite capacity of its resources ("finite scheduling" approaches). This system also underscored the potential benefits of distinguishing between bottleneck and non-bottleneck resources [15, 10]. In OPT, bottlenecks are scheduled first to optimize the throughput of the plant. Later, the production schedule is completed by compactly scheduling non-bottleneck operations to reduce inventory. The distinction between bottleneck and non-bottleneck machines was pushed one step further in the OPIS system [35,24], as it was recognized that new bottlenecks can appear during the constnuction of the schedule. The OPIS scheduler combines two scheduling perspectives: a resource-centered perspective for scheduling bottleneck resources, and a job-centered perspective to schedule non-bottleneck operations on a job-by-job basis. Rather than relying on its initial bottleneck analysis, OPIS typically repeats this analysis each time a resource or a job

\footnotetext{
'For instance, if an end-product required by the end of week 2 is obtained by assembling two sub-components and the assembly process typically takes a week to be completed, both sub-components will be required by the end of week 1.

${ }^{2}$ MRP systems are generally run on a weekly, possibly even a monthly basis.
} 
has been scheduled. This ability to detect the emergence of new bottlenecks during the construction of the schedule and revise the current scheduling strategy has been termed opportunistic scheduling [24]. Nevertheless, the opportunism in this approach remains limited in the sense that it typically requires scheduling an entire bottleneck (or at least a large chunk of it) before being able to switch to another one. For this reason, we actually refer to these techniques as macro-opportunistic.

In fact, variations in the job mix over time often cause different machines (or groups of machines) to be bottlenecks over different time intervals. Bottlenecks are sometimes said to "wander over time". Also, as a schedule is constructed for a bottleneck machine, a new machine can become more constraining than the original bottleneck. For instance, scheduling decisions on a bottleneck machine might require that a large number of jobs be processed on a preceding machine over a short period of time. At some point during the construction of the schedule, contention for the preceding machine might become higher than that for the original bottleneck. A scheduling technique that can only schedule large resource/job subproblems will not be able to take such considerations into account. It will overconstrain its set of alternatives before having worked on the subproblems that will most critically affect the quality of the entire schedule. This, in turn, will often result in poorer solutions. A more flexible approach would stop scheduling operations on a resource as soon as another resource is identified as more constraining. In the presence of multiple bottlenecks, such a technique would be able to shift attention from one bottleneck to another during the construction of the schedule rather than focus on the optimization of a single bottleneck at the expense of others. This paper presents such a flexible approach to scheduling. We call it micro-opportunistic scheduling. In this approach, resource contention is continuously monitored during the construction of the schedule, and the problem solving effort is constantly redirected toward the most serious bottleneck resource. In its simplest form, this micro-opportunistic approach results in an operation-centered view of scheduling, in which each operation is considered an independent decision point and can be scheduled without requiring that other operations using the same resource or belonging to the same job be scheduled at the same time ${ }^{3}$.

Experimental results presented at the end of this paper indicate that micro-opportunistic scheduling procedures often yield better schedules than less flexible bottleneck-centered approaches. Because of their flexibility, micro-opportunistic scheduling heuristics also seem particularly well suited to solving problems in which some operations have to be performed within non-relaxable time windows $[29,31]$ as well as repairing schedules in the face of contingencies. Finally, we find that they can easily be integrated in interactive systems in which

\footnotetext{
${ }^{3}$ An alternative approach in which resources can be resequenced to adjust for resource schedules built further down the road is described in [1] and [7]. This approach has been very successful at minimizing makespan, namely, the total duration of the schedule. This measure is closely related to the throughput of the plant but does not account for individual job due dates, tardiness costs or inventory costs. Attempts to generalize the procedure to account for due dates seem to have been less successful so far [34]. It should be pointed out that the idea of continuously reoptimizing the current partial schedule is compatible with a micro-opportunistic approach.
} 
manual and automatic scheduling decisions can be interleaved, thereby allowing the user to incrementally manipulate and compare alternative schedules (e.g., "What-if" type of analysis).

\subsection{Paper Outline}

The remainder of this paper successively reviews the predictive, reactive, and interactive capabilities of the Micro-Boss scheduling system.

Section 2 describes the micro-opportunistic search procedure implemented in Micro-Boss, focusing on look-ahead techniques used to measure contention, and heuristics to identify and schedule critical operations. A small example illustrating the use of these techniques is provided in Section 3. Section 4 describes the reactive and interactive components of the system. Section 5 reports the results of an experimental study comparing Micro-Boss with several popular scheduling approaches, including coarser opportunistic schedulers, under a wide range of simulated situations. Finally, Section 6 briefly reviews current research efforts and summarizes the impact of this work. 


\section{A Micro-opportunistic Search Procedure}

In this section, a deterministic scheduling model is assumed, in which all jobs to be scheduled are known in advance. Issues pertaining to reactive scheduling and control in the face of manufacturing contingencies such as machine breakdowns are addressed in a later section.

\subsection{A Deterministic Scheduling Model}

For the time being, we consider a deterministic scheduling problem in which a set of jobs $J=\left\{j_{1}, \ldots j_{n}\right\}$ has to be scheduled on a set of physical resources $R E S=\left\{R_{1}, \ldots, R_{m}\right\}$. Each job $j_{l}$ consists of a set of operations $O^{l}=\left\{O_{1}^{l}, \ldots, O_{n_{l}}^{l}\right\}$ to be scheduled according to a process routing that specifies a partial ordering among these operations (e.g., $O_{i}^{l}$ BEFORE $O_{j}^{l}$ ). We further assume scheduling problems with in-tree process routings, namely process routings in which operations can have one or several direct predecessors but at most one direct successor (e.g., assembly process routings). This is by far the most common type of process routing encountered in manufacturing.

Additionally, each job $j_{l}$ has an earliest acceptable release date, $e r d_{l}$, a due-date, $d d_{l}$, and a latest acceptable completion date, $l c d_{l}$, where $l c d_{l} \geq d d_{l} \geq e r d_{l}$. All jobs need to be scheduled between their earliest acceptable release date and latest acceptable completion date ${ }^{4}$. The earliest acceptable release date might correspond to the earliest possible arrival date of raw materials. It is assumed that the actual release date (or job start date) will be determined by the schedule that is constructed. The latest acceptable completion date might correspond to a date after which the customer will refuse delivery. If such a date does not actually exist, it can always be chosen far enough in the future so that it is no longer a constraint.

Each operation $O_{i}^{t}$ has an expected duration, $d u_{i}^{l}$, and a start time, $s t_{i}^{l}$ (to be determined), whose domain of possible values is delimited by an earliest start time, est $t_{i}$, and a latest start time, $i s t_{i}^{t_{i}}$ (initially derived from the job's earliest acceptable release date $\operatorname{erd}_{l}$ and latest acceptable completion date $\left.l c d_{l}\right)$. We assume that each operation $O_{i}^{l}$ requires a single resource $R_{i}^{l}$ for which there might be several alternatives in RES. The model further allows for resource availability constraints that specify the times when each resource is normally available (e.g., what the number of shifts is and whether the resource is available over the week-end). Finally, setup operations might be required before an operation can start on a machine. Examples of setup operations include changing the fixtures holding a part, loading a new part, cleaning a painting station when switching from one color to another, etc.

The objective of the scheduling system, under deterministic assumptions, is to build a schedule that satisfies the above constraints and minimizes (as much as possible) the costs incurred for missing due dates or carrying overhead inventories. These costs are briefly described below.

\section{$\underline{\cos T s}$}

\footnotetext{
${ }^{4}$ Notice that this formulation does not exclude infeasible problems.
} 
Each job $j_{l}$ has

- A marginal tardiness cost, tard $l$ This is the cost incurred for each unit of time that the job is tardy (i.e., finishes past its due date). Marginal tardiness costs generally include tardiness penalties, interest on delayed profits, loss of customer goodwill, etc ${ }^{5}$. The tardiness cost of job $j_{l}$ in a given schedule is

$$
T A R D_{l}=\operatorname{tard}_{l} \times \operatorname{Max}\left(0, C_{l}-d d_{l}\right)
$$

where $C_{l}=s t_{n_{l}}^{l}+d u_{n_{l}}^{l}$ is the completion date of job $j_{l}$ in that schedule, assuming that $O_{n_{l}}^{l}$ is the last operation in job $j_{l}$.

- Marginal in-process and finished-goods inventory costs: In our model, each operation $O_{i}^{l}$ can incrementally introduce its own non-negative marginal inventory cost, inv $v_{i}^{l}$. Typically, the first operation in a job introduces marginal inventory costs that correspond to interest on the costs of raw materials, interest on processing costs (for that first operation), and marginal holding costs. Downstream operations ${ }^{6}$ introduce additional marginal inventory costs such as interest on processing costs or interest on the costs of additional raw materials required by these operations. The total inventory cost for a job $j_{l}$, in a given schedule, is:

$$
I N V_{l}=\sum_{i=1}^{n_{l}} i n v_{i}^{l} \times\left[\operatorname{Max}\left(C_{l}, d d_{l}\right)-s t_{i}^{l}\right]
$$

This cost accounts for both work-in-process and finished-goods inventory costs ${ }^{7}$

The total cost of a schedule is obtained by summing the cost of each job schedule:

$$
\text { Schedule Cost }=\sum_{i=1}^{n}\left(T A R D_{l}+I N V_{l}\right)
$$

\section{A SMALL EXAMPLE}

Figure 1 depicts a small scheduling problem with four jobs that will be used in this section to illustrate the behavior of the micro-opportunistic scheduling heuristics implemented in MicroBoss. Each square box represents an operation and is labeled by the name of this operation (e.g.,

\footnotetext{
${ }^{5}$ In this model, inventory costs incurred after the due date are not included in the tardiness costs but, rather, in the inventory costs described below.

${ }^{6}$ An operation $O_{i}^{k}$ is said to be downstream (upstream) of another operation $O_{j}^{k}$ within its job if $O_{i}^{k}$ is a direct or indirect successor (predecessor) of $O_{j}^{k}$ in that job, as defined by the job's process routing.

${ }^{3}$ Note that, in this deterministic model, minimizing work-in-process inventory costs is equivalent to minimizing job leadtimes or flowtimes.
} 
$O_{1}^{1}$ ), its (expected) duration (e.g., $d u_{1}^{1}=2$ ), and the resource it requires (e.g., $R_{1}^{1}=R_{1}$ ). In this simple example, each operation is assumed to require a single resource, for which there are no substitutes. The arrows represent precedence constraints. For instance, job $j_{1}$ has 5 operations $O_{1}^{1}, O_{2}^{1}, \ldots, O_{5}^{1}$. $O_{1}^{1}$ has to be performed before $O_{2}^{1}, O_{2}^{1}$ before $O_{4}^{1}$, etc. The other arcs in the graph represent capacity constraints that require that each resource be allocated to only one operation at a time. There is a capacity constraint between each pair of operations that require the same resource. Notice that $R_{2}$ is the only resource required by four operations (one from each job). Notice also that in three out of four jobs (namely, $j_{1}, j_{3}$, and $j_{4}$ ), the operation requiring $R_{2}$ is one of the job's longest operations. Consequently, resource $R_{2}$ can be expected to be the main bottleneck of the problem. We will see that, to some extent, resource $R_{1}$ constitutes a secondary bottleneck.

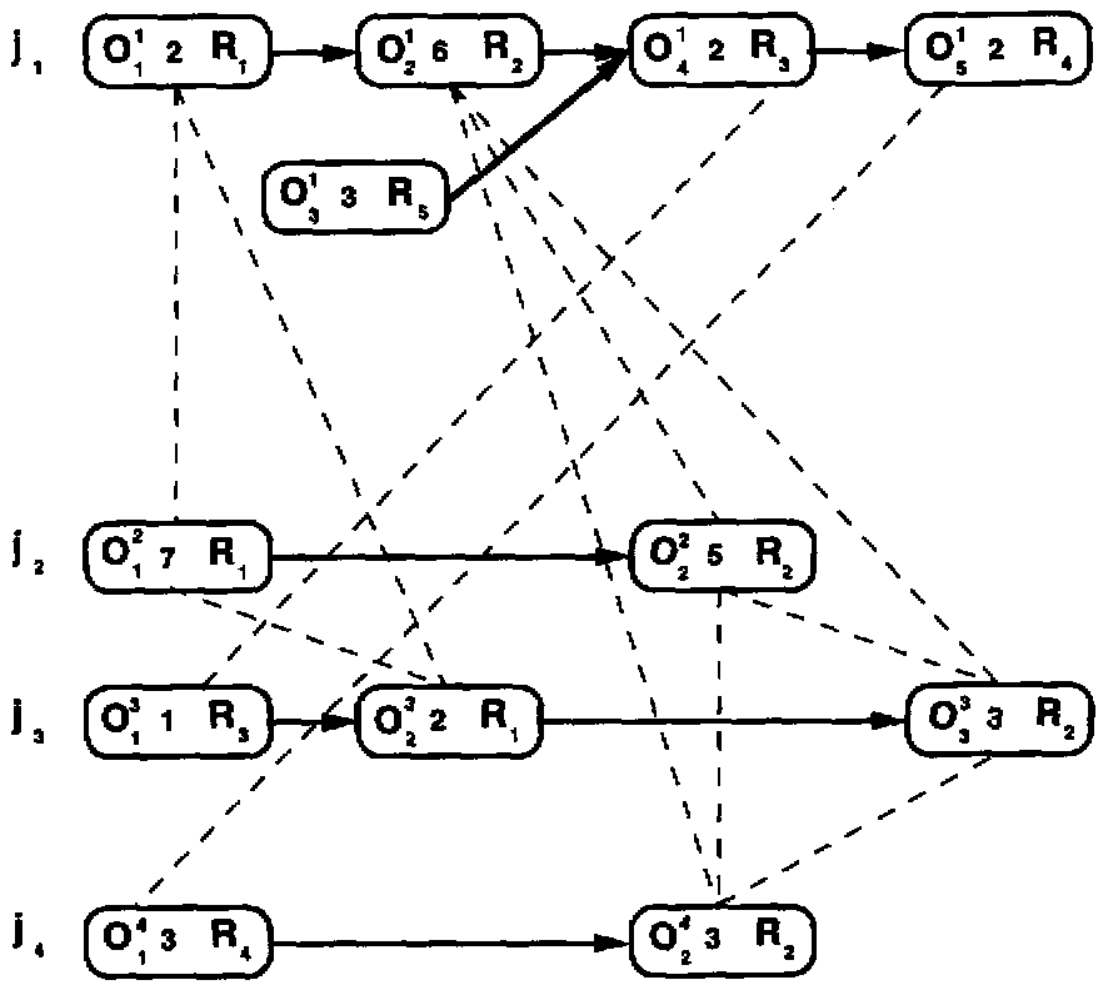

precedence constraint

- . . . . capacity constraint

Figure 1: A simple job shop problem with four jobs. Each node is labeled by the operation that it represents, its duration, and the resource that it requires.

The earliest acceptable release dates, due dates, and latest acceptable completion dates of the jobs are provided in Table 1 along with the marginal tardiness and inventory costs of these jobs. 
Earliest acceptable release dates, due dates, latest acceptable completion dates, and costs

\begin{tabular}{|c|c|c|c|c|c|c|c|c|c|}
\hline $\mathrm{Job} j_{l}$ & $e r d_{l}$ & $d d_{l}$ & $i c d_{l}$ & tard & inv $v_{1}^{l}$ & inv & inv $v_{3}^{l}$ & inv $v_{4}^{l}$ & inv $v_{5}^{l}$ \\
\hline$j_{1}$ & 0 & 12 & 20 & 20 & 2 & 1 & 2 & 0 & 0 \\
\hline$j_{2}$ & 0 & 14 & 20 & 20 & 5 & 0 & - & - & - \\
\hline$j_{3}$ & 0 & 9 & 20 & 5 & 1 & 0 & 0 & - & - \\
\hline$j_{4}$ & 0 & 18 & 20 & 10 & 1 & 0 & - & - & - \\
\hline
\end{tabular}

Table 1: Earliest acceptable release dates, due dates, latest acceptable completion dates and marginal costs.

\subsection{Overview of the Search Procedure}

In Micro-Boss, each operation is considered an independent decision point. Any operation can be scheduled at any time, if deemed appropriate by the system. There is no obligation to simultaneously schedule other operations upstream or downstream within the same job, nor is there any obligation to schedule other operations competing for the same resource.

Micro-Boss proceeds by iteratively selecting an operation to be scheduled and a reservation (i.e., a resource/time interval) to be assigned to that operation. Every time an operation is scheduled, a new search state is created, where new constraints are added to account for the reservation assigned to that operation. A consistency enforcing procedure then takes care of updating the set of remaining possible reservations of each unscheduled operation. If an unscheduled operation is found to have no possible reservations left, a deadend state has been reached, in which case the system needs to backtrack (i.e., it needs to undo some earlier reservation assignments to be able to complete the schedule). If the search state does not appear to be a deadend, the system moves on and looks for a new operation to schedule and a reservation to assign to that operation.

To enhance search efficiency ${ }^{8}$ and produce high quality schedules, Micro-Boss interleaves search with the application of consistency enforcing mechanisms and a set of look-ahead techniques that help decide which operation to schedule next (operation ordering heuristic) and which reservation to assign to that operation (reservation ordering heuristic).

1. Consistency Enforcing/Checking: Consistency enforcing techniques prune the search space by inferring new constraints resulting from earlier reservation assignments $[19,30]$. By constantly accounting for earlier scheduling decisions, these techniques reduce the chances of reaching a deadend (i.e., a partial schedule

\footnotetext{
${ }^{8}$ We define search efficiency as the ratio of the number of operations to be scheduled over the number of search states generated. If the number of search states generated to build the schedule is equal to the number of operations, search efficiency is equal to 1.
} 
that cannot be completed without backtracking). Simultaneously, by allowing for the early detection of deadend states, these techniques limit the amount of work wasted in the exploration of fruitless alternatives.

2. Look-Ahead Analysis: A two-step look-ahead procedure is applied in each search state, which first optimizes reservation assignments within each job and then, for each resource, computes contention between jobs over time. Resource/time intervals where contention is the highest help identify the critical operation to be scheduled next (operation ordering heuristic). Reservations for that operation are then ranked according to their ability to minimize the costs incurred by the jobs contending for the critical resource (reservation ordering heuristic). By constantly redirecting its effort toward the most serious conflicts, the system is able to build schedules that are closer to the global optimum. Simultaneously, because the scheduling strategy is aimed at reducing job contention as rapidly as possible, chances of reaching deadend states tend to quickly subside too.

The opportunism in Micro-Boss results from the ability of the system to constantly revise its search strategy and redirect its effort toward the scheduling of the operation that appears to be the most critical in the current search state. This degree of opportunism differs from the one displayed by earlier approaches where scheduling entities were large resource/job subproblems $[24,6]$, i.e., where large resource/job subproblems had to be scheduled before the system could revise its scheduling strategy.

Concretely, given a scheduling problem such as the one described in Figure 1, Micro-Boss starts in a search state in which no operation has been scheduled yet ${ }^{9}$, and proceeds according to the following steps:

1. If all operations have been scheduled, then stop; else go on to 2 .

2. Apply the consistency enforcing procedure.

3. If a deadend is detected then backtrack; else go on to 4.

4. If one or more operations were found to have only one possible reservation left, then schedule these operations (creating a new search state for each one). If all operations have been scheduled, then stop; else go on to 5.

\footnotetext{
${ }^{9}$ Alternatively, Micro-Boss can also complete a partial schedule, in which case the initial search state corresponds to the initial partial schedule. A description of reactive and interactive capabilities of the system is provided in Section 4.
} 
5. Perform a look-ahead analysis: Rank the possible reservations of each unscheduled operation according to how well they minimize the costs of the job to which the operation belongs (step 1), and evaluate resource contention over time (step 2).

6. Select the next operation to be scheduled (i.e., operation ordering heuristic).

7. Select a reservation for that operation (i.e., reservation ordering heuristic).

8. Create a new search state by adding the new reservation assignment to the current partial schedule. Go back to 1 .

As in other constraint-directed scheduling systems [16], the consistency enforcing procedure used in Micro-Boss (1) maintains for each unscheduled operation a pair of earliest/latest possible start times and (2) marks as unavailable those resource/time intervals allocated to already scheduled operations. Additionally, reservation pruning performed by the Micro-Boss consistency procedure also accounts for resource/time intervals that are absolutely needed by unscheduled operations. Figure 2 displays an example of an unscheduled operation $O_{i}^{k}$ whose earliest and latest possible reservations overlap. Whichever reservation this operation is ultimately assigned, it will always need time interval $\left[l s t_{i}^{k}, e f_{i}^{k}\right]$. Accordingly, the Micro-Boss consistency procedure prunes the set of remaining possible reservations of other unscheduled operations requiring that resource by removing all those reservations that overlap with time interval $\left[l s t_{i}^{k}, e f f_{i}^{k}\right]^{10}$.

Results presented in this paper were obtained using a simple chronological backtracking scheme. Experimentation with more sophisticated backtracking schemes is described in [31].

The remainder of this section gives a more detailed description of the look-ahead analysis and the operation/reservation ordering heuristics used in Micro-Boss. Further details on these techniques, as well as other aspects of the system, can be found in [30].

\footnotetext{
${ }^{10}$ This differs from an earlier version of the system [30], in which resourceftime intervals needed by unscheduled operations were only used to detect conflicts. In this earlier version, a conflict would be detected when two or more unscheduled operations needed overlapping resource/time intervals. Rather than waiting for such conflicts to arise, our new consistency procedure efficiently prevents such conflicts from occurring, thereby further reducing backtracking. A generalized version of this procedure is used for parallel machines.
} 


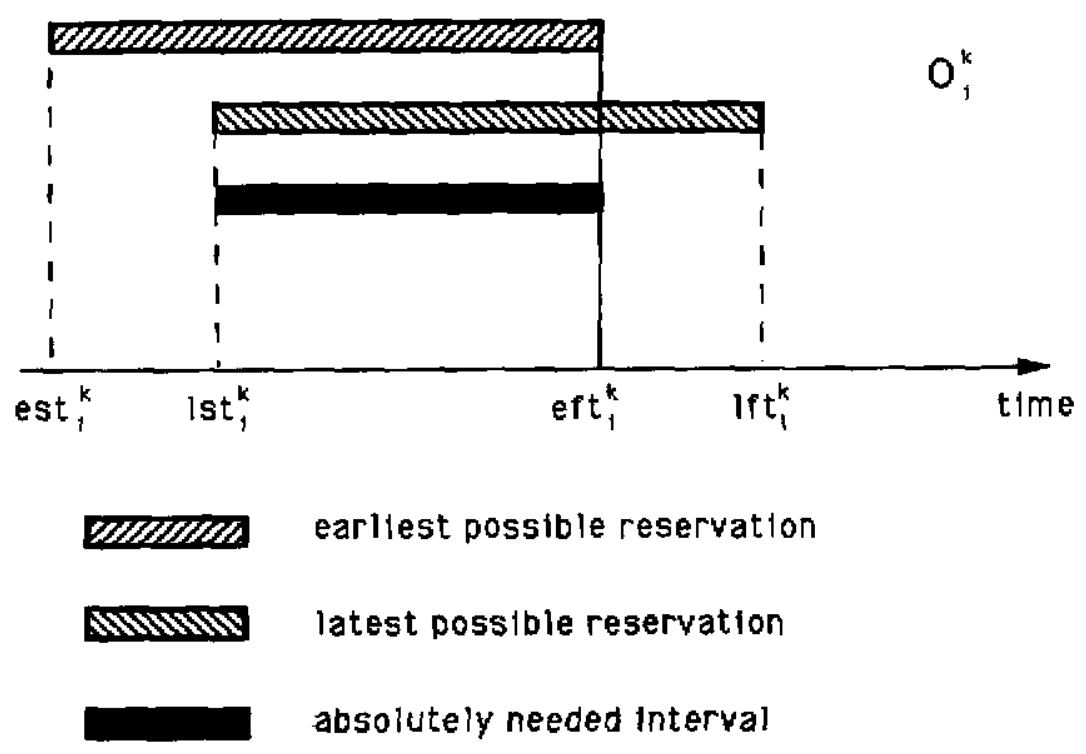

Figure 2: An example of an unscheduled operation that absolutely needs a resource/time interval.

\subsection{Look-Ahead Analysis in Micro-Boss}

\subsubsection{Optimizing Critical Conficts First}

If all jobs could be scheduled optimally (i.e., just-in-time), there would be no scheduling problem. Generally, this is not the case. Jobs typically have conflicting resource requirements. The look-ahead analysis carried out by Micro-Boss in each search state aims at helping the scheduling system focus its effort on those conflicts that currently appear most critical. A critical conflict is one that will require an important trade-off, i.e., a trade-off that will significantly impact the quality of the entire schedule. By first focusing on critical conflicts, Micro-Boss ensures that it has as many options as possible to optimize these conflicts. As illustrated by a trace provided in the next section, once critical trade-offs have been worked out, the remaining unscheduled operations tend to become more decoupled and, hence, easier to optimize $^{11}$. As contention subsides, so does the chance of needing to backtrack. In other words, by constantly redirecting search towards those trade-offs that appear most critical, Micro-Boss is expected to produce better schedules and simultaneously keep backtracking at a low level.

More specifically, a two-step look-ahead procedure is applied to each search state. This procedure first optimizes reservation assignments within each job and then, for each resource, computes contention between jobs over time. The so-called demand profiles produced by these computations help identify operations whose good reservations (as identified in the first step) conflict with the good reservations of other operations. These operations define the critical

\footnotetext{
${ }^{11}$ This is similar to the way bottleneck schedules drive other scheduling decisions in OPT.
} 
conflicts on which Micro-Boss works first.

This two-step look-ahead analysis is further detailed below.

\subsubsection{Step 1: Reservation Optimization within a Job}

In order to measure contention between the resource requirements of unscheduled operations, Micro-Boss keeps track of the best start times that remain available to each unscheduled operation within its job. Additionally, the system implicitly maintains, for each remaining possible start time $\tau$ of each unscheduled operation $O_{i}^{k}$, a function mincost ${ }_{i}^{k}(\tau)$ that indicates the minimum additional costs that would be incurred by job $j_{k}$ (the job to which $O_{i}^{k}$ belongs), if $O_{i}^{k}$ were to start at $s t_{i}^{k}=\tau$ rather than at one of its best possible start times. By definition, if $s t_{i}^{k}=\tau$ is one of the best start times that remain available to $O_{i}^{k}$ within its job, then $\operatorname{mincost}_{i}^{k}(\tau)=0$. Rather than explicitly maintaining mincost functions, Micro-Boss simply maintains for each unscheduled operation $O_{i}^{k}(1)$ an apparent marginal tardiness cost, app-tard ${ }_{i}^{*}$, that approximates the cost incurred by job $j_{k}$ for each unit of time that $O_{i}^{k}$ starts past its latest best start time and (2) an apparent marginal inventory cost, app-inv $v_{i}^{k}$, that approximates the cost incurred by job $j_{k}$ for each unit of time that $\alpha_{i}^{k}$ starts before its earliest best start time. These costs are updated in each search state to account for earlier scheduling decisions, using a set of efficient propagation procedures described in [30].

\subsubsection{Step 2: Building Demand Profiles to Identify Critical Resource/Time Intervals}

In Micro-Boss, critical conflicts are identified as groups of operations whose good reservations (within their jobs) conflict with each other. The importance of a conflict depends on the number of operations that are competing for the same resource, the amount of temporal overlap between the requirements of these operations, the number of alternative reservations still available to each of these conflicting operations and their costs, as determined by the mincost functions computed in step 1 .

To identify critical conflicts, Micro-Boss uses a probabilistic framework in which each remaining possible start time $\tau$ of an unscheduled operation $\sigma_{i}^{\prime}$ is assigned a subjective probability $\sigma_{i}^{l}(\tau)$ to be selected for that operation in the final schedule. Possible start times with lower mincost values are assigned a larger probability, thereby reflecting our expectation that they will yield better schedules. Given these start time probability distributions, the probability that an unscheduled operation $O_{i}^{l}$ uses its resource ${ }^{12}$ at time $t$, which is referred to as the individual demand of $O_{i}^{l}$ for $R_{i}^{d}$, is:

\footnotetext{
${ }^{12}$ For the sake of simplicity, we assume here that each operation requires a single resource for which there are no alternatives. The construction of demand profiles can easily be generalized to deal with parallel machines by building profiles for entire groups of machines and normalizing them based on their remaining available capacities over time.
} 


$$
D_{i}^{l}(t)=\sum_{t-d u_{i}<\tau \leq t} \sigma_{i}^{l}(\tau)
$$

where $d u_{i}^{l}$ is the duration of $O_{i}^{l}, D_{i}^{l}(t)$ is also a (subjective) measure of the reliance of operation $O_{i}^{l}$ on the availability of its resource at time $t$. By adding the individual demands of all unscheduled operations requiring a given resource, say $R_{k}$, the system obtains an aggregate demand profile, $D_{R_{x}}^{a g g r}(t)$, that indicates contention between (all) unscheduled operations for that resource $R_{k}$ as a function of time:

$$
D_{R_{k}}^{a g g r}(t)=\sum D_{i}^{l}(t)
$$

where the summation is carried over all unscheduled operations that need resource $R_{k}$.

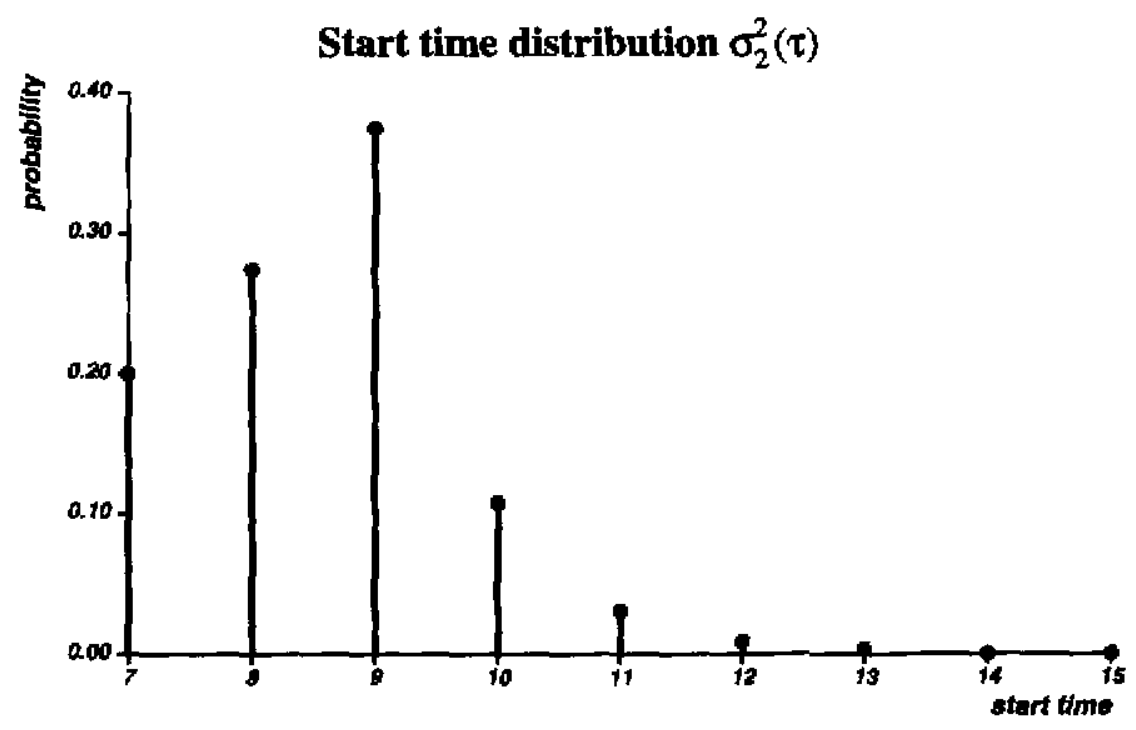

Figure 3: Start time distribution $\sigma_{2}^{2}(\tau)$ for operation $O_{2}^{2}$ in the initial search state for the problem defined in Figure 1.

Figure 3 displays $\sigma_{2}^{2}(\tau)$, the start time distribution of operation $o_{2}^{2}$ in the problem defined in Figure 1. This start time distribution is depicted in the initial search state, where all operations still have to be scheduled. In this search state, start time $s t_{2}^{2}=9$ is the best possible start time for $O_{2}^{2}$ : it corresponds to a just-in-time schedule of job $j_{2}$. Later start times have a lower subjective probability because they would force the job to finish after its due date. Earlier start times are also suboptimal because they would produce additional inventory. In this example, the marginal tardiness cost of job $j_{2}$, $\operatorname{tard}_{2}=20$, is four times larger than the marginal inventory cost introduced by operation $O_{1}^{2}$, in $v_{1}^{2}=5$. Accordingly, $\sigma_{2}^{2}(\tau)$ decreases faster for $\tau>9$ than for $\tau<9$. 

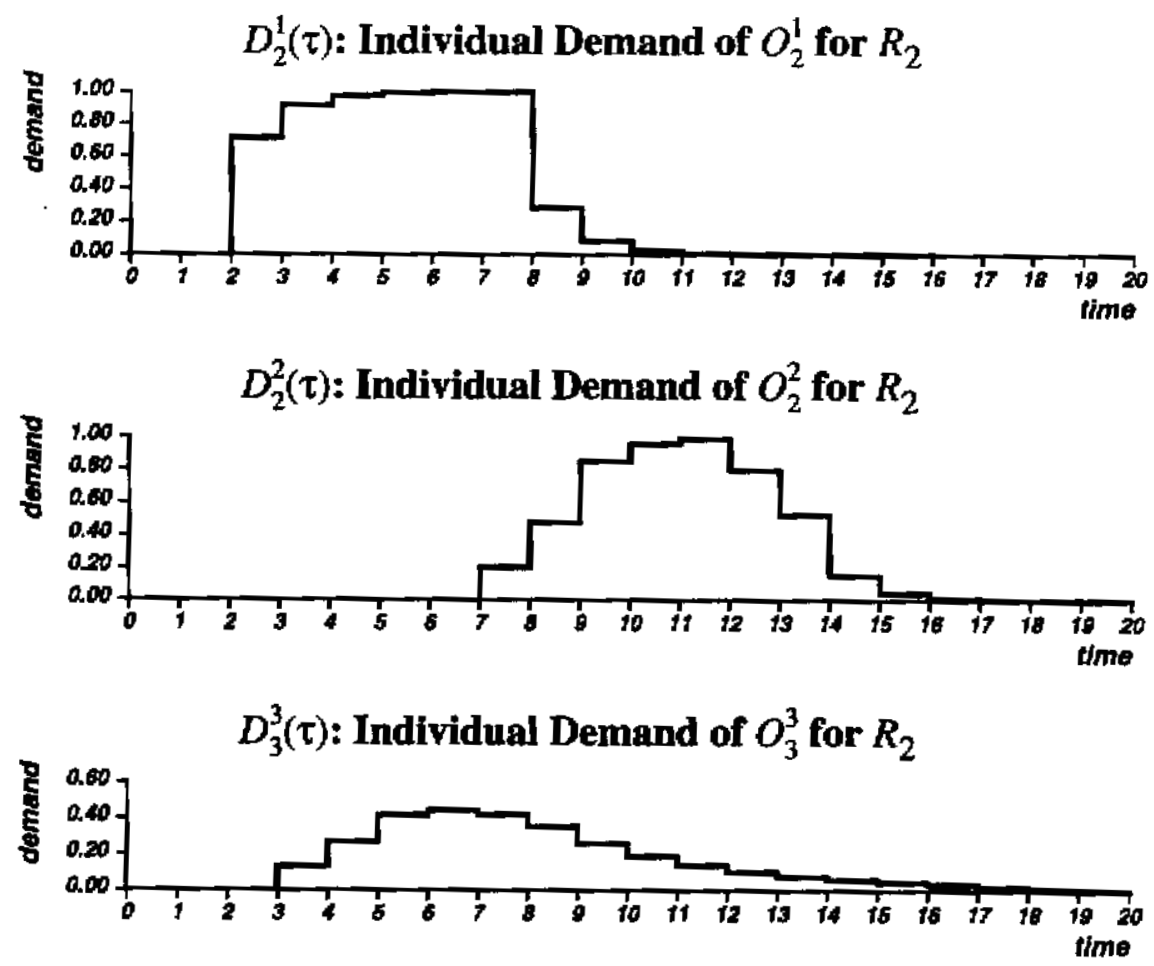

$D_{2}^{4}(\tau):$ Individual Demand of $O_{2}^{4}$ for $R_{2}$

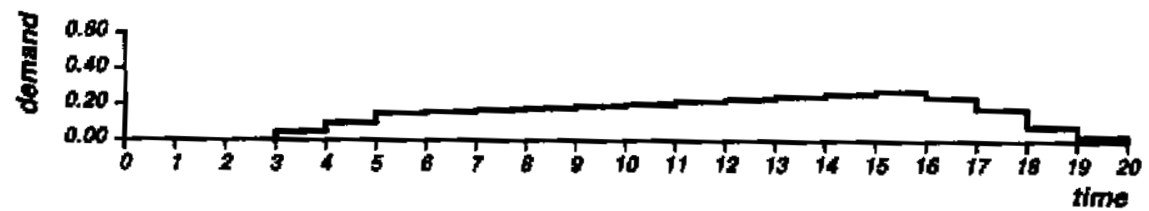

$D_{R_{2}}^{a g g r}(\tau):$ Aggregate Demand for $R_{2}$

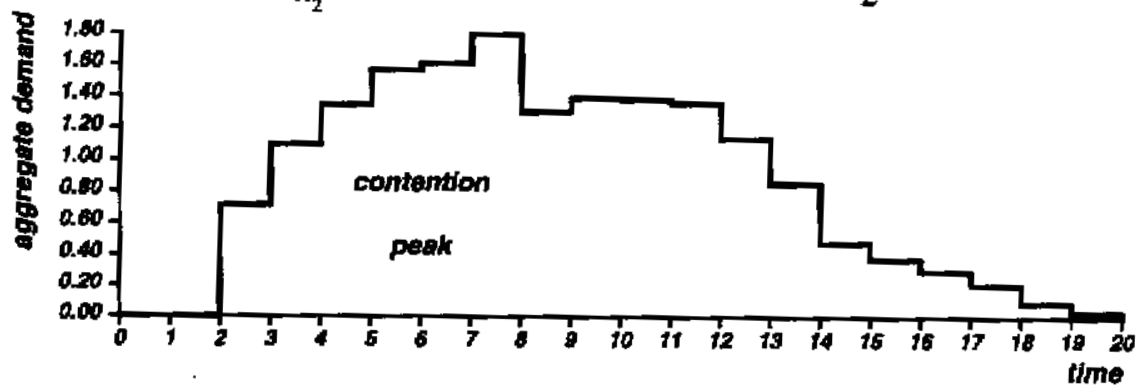

Figure 4: Building $R_{2}$ 's aggregate demand profile in the initial search state.

Figure 4 displays the individual demand profiles of the four operations requiring resource $R_{2}$. These demand profiles represent the subjective probability that each one of these operations uses resource $R_{2}$ as a function of time. The aggregate demand for resource $R_{2}$ is obtained by summing 
these four individual demands over time. The individual demands of operations $O_{3}^{3}$ and $O_{2}^{4}$ are quite uniform because these two operations have relatively low apparent marginal costs (see the marginal tardiness and inventory costs of job $j_{3}$ and job $j_{4}$ in Table 1). In contrast, operations $O_{2}^{1}$ and $o_{2}^{2}$, which have larger apparent marginal costs, have individual demands that are concentrated around their best reservations.

Similar computations can be performed for each of the five resources in the problem. The resulting aggregate demands (in the initial search state) are displayed in Figure 5 . As expected, resource $R_{2}$ appears to be the most contended for. The aggregate demand for that resource is well above 1.0 over a large time interval, with a peak at 1.79 . Resource $R_{1}$ appears to be a potential bottleneck at the beginning of the problem, with a demand peaking at 1.52 . Whether $R_{1}$ will actually be an auxiliary bottleneck or not cannot be determined directly from the curves displayed in Figure 5. Instead, the system needs to update these curves in each search state to account for earlier decisions. It could be the case that as operations requiring $R_{2}$ are scheduled, the aggregate demand for $R_{1}$ becomes smoother. In this example, this is not the case. On the contrary, as operations are scheduled on resource $R_{2}$, some operations on resource $R_{1}$ end up with only one possible reservation and need to be immediately scheduled, as indicated by the trace provided in Section 4.

\subsection{Operation Selection}

Critical operations are identified as operations whose good reservations (as identified in the first step of the look-ahead analysis) conflict with the good reservations of other operations. The largest peak in the aggregate demand profiles determines the next conflict (or micro-bottleneck) to be optimized; the operation with the largest reliance on the availability of the corresponding resource/time interval (i.e., the operation with the largest individual contribution to the peak) is selected to be scheduled next. Indeed, this operation is the one whose good reservations are the most likely to become unavailable if other operations contending for the current microbottleneck were scheduled first.

In the example introduced earlier, the largest demand peak is the one for resource $R_{2}$ over interval [4,8[. Figure 6 displays the aggregate demand for resource $R_{2}$ together with the individual demands of the four operations requiring this resource. The operation with the largest contribution to the demand peak is $O_{2}^{1}$. Therefore this operation is selected to be scheduled next. This is no real surprise: $O_{2}^{1}$ belongs to one of the two jobs in the problem that have a high marginal tardiness cost $\left(\operatorname{tar} d_{1}=20\right)$. While any delay in starting job $j_{1}$ will result in large tardiness costs, job $j_{3}$ (i.e., the job with the next highest contribution) can tolerate a small delay and is subject to lower tardiness penalties.

The computation of demand profiles, as described in 2.3.3 can be quite expensive when performed for each resource in each search state over the entire scheduling horizon. Micro-Boss can avoid this problem by incrementally maintaining a set of rough demand profiles for each resource (or group of identical resources). These rough demand profiles use a much coarser time 

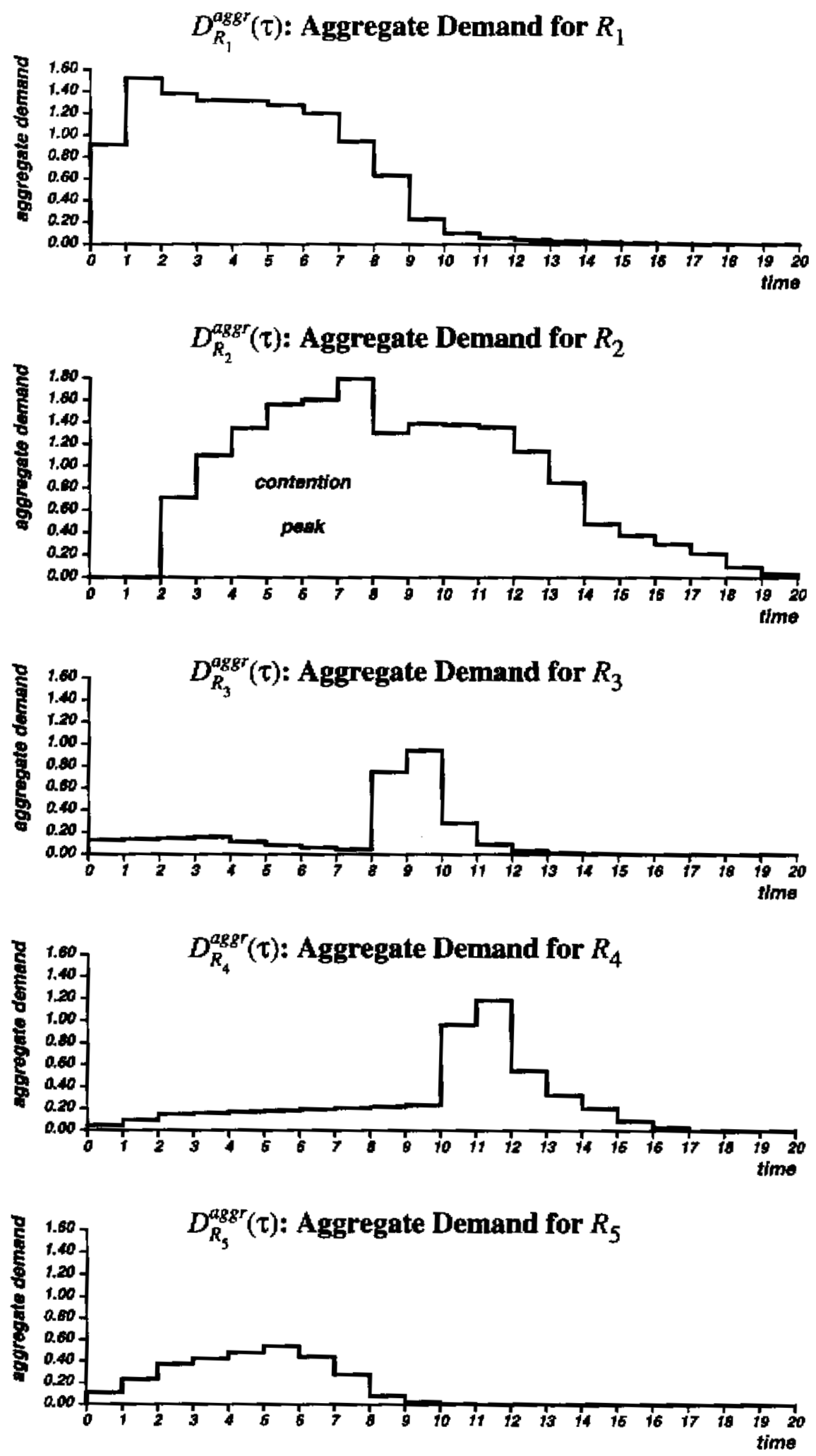

Figure 5: Aggregate demands in the initial search state for each of the five resources. 

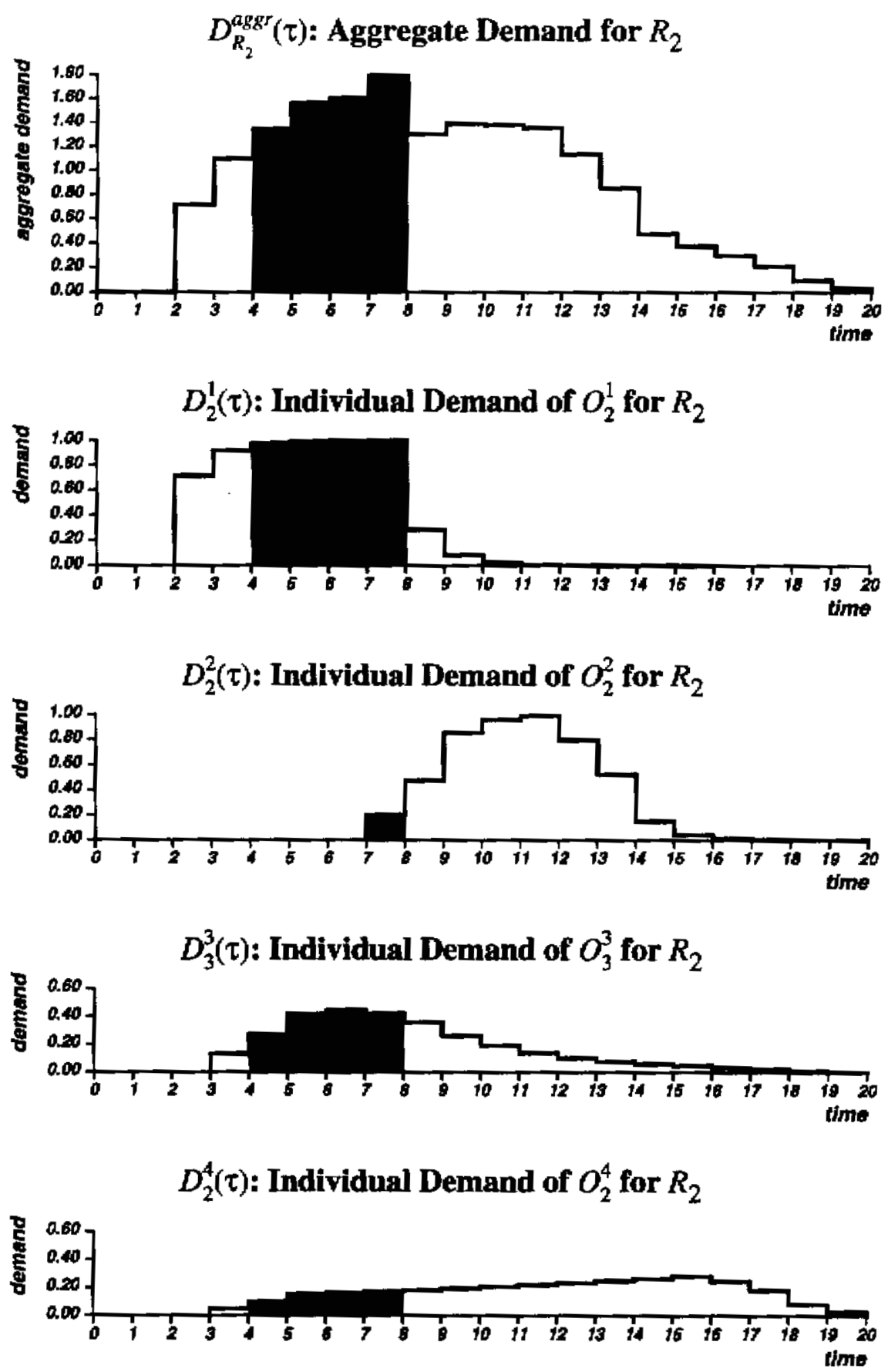

Figure 6: Operation selection in the initial search state.

granularity and are obtained by splitting the demand of each unscheduled operation into two 
components. One component ( $50 \%$ of the operation's total demand in the current implementation 13 ) is evenly spread between the start and end times of the latest best reservation of the operation while the second component (the remaining $50 \%$ of the operation's demand) is evenly spread between the earliest start time and latest finish time of the operation. Rough demand profiles can be quickly updated as the system moves from one search state to the next and are used in each search state to identify a small number of critical resource/time intervals over which the more detailed demand profiles described in 2.3 .3 are then constructed.

\subsection{Reservation Selection}

To schedule the critical operation identified in 2.4 , the system attempts to identify a reservation (for the critical operation) that will reduce as much as possible the costs incurred by the job to which that operation belongs and the other jobs with which that operation competes. This is approximated as a single-machine or parallel-machine early/tardy scheduling problem in which operations scheduled past their best start times incur penalties determined by their apparent marginal tardiness costs, while operations scheduled before their best start times incur earliness penalties, as determined by their apparent marginal inventory costs $[2,30]$. In the experiments reported at the end of this paper, several variations of a single-machine early/tardy procedure developed by $\mathrm{Ow}$ and Morton $[26,30]$ were successively run and the single-machine schedule with the lowest cost was used to determine the reservation assigned to the critical operation. More recently, a new scheduling heuristic has also been developed to solve problems with setups [17].

${ }^{13}$ The total demand of an operation is equal to its duration. 


\section{A Small Example}

Micro-Boss is implemented in $\mathrm{C}++$ with an $\mathrm{X}^{\mathrm{TM}} /$ Motif $^{\mathrm{TM}}$ interface. The small example used throughout this paper requires less than $0.1 \mathrm{CPU}$ seconds on a DECstation ${ }^{\mathrm{TM}} 5000 / 200$ running under UNIX ${ }^{\mathrm{TM} 14}$. An edited trace of this example is given in Figure 7.

In this example, the scheduling procedure first focuses on the scheduling of the main bottleneck resource, $R_{2}$. However, as it schedules operations on this resource, the system can also jump to other resources and consolidate the schedule by allocating reservations to critical operations requiring these other resources. In this small example where operations have a small number of possible reservations, this is mainly accomplished through the identification of operations that have only one possible reservation left (e.g. the scheduling of $O_{1}^{1}$ or $O_{1}^{2}$ ). In general, this can be done based on the contention analysis performed by Micro-Boss (e.g., the identification of a critical conflict on resource $R_{4}$ at depth 6). As a result, the system jumps back and forth between several resources, always trying to focus on what appears to be the most critical decision.

The average expected demand displayed in each search state is the average demand for the critical demand peak, and the average contribution is the percentage of the total demand for the peak that comes from the critical operation. When search starts, contention is relatively high, as illustrated by the average expected demand for the critical peak (1.58 at depth $0,1.73$ at depth 2 and 1.50 at depth 4) and the relatively low contribution of the critical operation to the demand for the peak (e.g., $O_{2}^{1}$ contributes only $63 \%$ of the total demand for the peak in the initial search state, $O_{2}^{2} 57 \%$ at depth 2, etc.) indicating that the resource requirements of the critical operation compete with those of several other operations. During construction of the schedule, the average demand for the critical peak progressively decreases ${ }^{15}$ and the critical operation progressively contributes a larger percentage of the demand for the critical peak. This indicates that contention between unscheduled operations decreases. After half of the operations have been scheduled (depth 7), contention has totally disappeared: the critical operation is the only one to contribute to the demand for the peak. The resource requirements of the operations that still need to be scheduled no longer compete with each other. This is not particular to this example: the same has been observed on all the problems we have nu and suggests that the operation ordering heuristic implemented in Micro-Boss is indeed very effective at redirecting search towards the most serious conflicts.

Notice also that no backtracking was necessary to schedule this problem. The resulting schedule is displayed in Figure 8.

\footnotetext{
${ }^{14} \mathrm{X}$ Window System is a registered trademark of the Massachusetts Institute of Technology. Motif is a registered trademark of the Open Software Foundation, Inc. UNIX is a registered trademark of UNIX Systems Laboratories, Inc. DECstation is a registered trademark of the Digital Equipment Corporation.
}

${ }^{15}$ Remember that the demand peak corresponds to the interval of highest contention in the current search state. 
> Depth: 0, Number of atater visited: 0

Critical demand peak:

$R_{2}$ between 4 and 8 , Avg. expected demand: 1.58

Cxitical operation: $O_{2}^{\prime}$, Arg. contrib.: 63\%

$O_{2}^{1}$ is locheduled between 2 and $\mathrm{a}$ on $R_{2}$

>> Depth: 1, Number of atates viaited: 1

$O_{1}^{1}$ hes only one poselble reservation left

and is acheduled between 0 and 2 on $R_{1}$

>> Depth: 2, knmber of states visited: 2

Crttical demand peak:

$R_{2}$ batween 10 and 14, Avg. expocted derand: 1.73

Critical operation: $o_{2}^{2}$, Avg. contrib.: 57\%

$O_{2}^{2}$ is schectuled between 9 and 14 on $R_{2}$

> Depth: 3, Mumber of wtetes visited: 3

$O_{1}^{2}$ has only one poesible reservation left

and is schedulud between 2 and 9 on $R_{1}$

>> Dopth: 4, Mumber of statea riatted: 4

Critical demand poak:

$R_{2}$ between 14 and 18, Avg. woseted demand: 1.50

Critical operation: $O_{2}^{4}$, Avg. contrib.: 50\%

$o_{2}^{4}$ is mcheduled botween 14 and 17 on $R_{2}$

>> Depth: 5, Number of states visited: 5

$O_{3}^{3}$ has only one poseible reservation lett

and is acheduled betwen 17 and 20 on $R_{2}$

> Dupth: 6, Humber of atates visited: 6

Critical demand paks:

$R_{4}$ botwen 10 and 12, $\mathrm{N} v$. expected demand: 1.12

Critleal operation: $o_{5}^{l}$, Avo. contrtb.: 73\%

$O_{5}^{1} 1$ acheduled betwoen 20 and 12 on $R_{4}$

> Depth: 7, Nuber of etates vinited: 7

$O_{4}^{1}$ has only one poastble reaervation left

and 1 a echeduled betwen 8 and 10 on $R_{3}$

Figure 7: An edited trace 
>> Depth: 8, Number of states visited: 8 Critical domand peak:

$R_{5}$ between 5 and 8, Avg. expected demand: 0.95 Critical operation: $O_{3}^{1}$, Avg. contrib.: 100\% $o_{3}^{1}$ is scheduled batreen 5 and $B$ on $R_{5}$

> Depth: 9, Number of atates visited: 9 Critical demand poak: $R_{4}$ between 7 and 9, Arg. expected demend: 0.96 critical operation: $O_{1}^{4}$, nog. contrtb. : 100\% $O_{1}^{4}$ is scheduled batween 7 and 10 on $R_{4}$

>> Depth: 10, Number of states visited: 10 Criticel demand peak:

$R_{1}$ botween 14 and 17 , Arg. axpected dumand: 0.65 Critical operation: $O_{2}^{3}$, Avg contrib.: 100\% $O_{2}^{3}$ 1s scheduled betwen 15 and 17 on $R_{1}$

>> Depthi 11, Number of gtates risited: 11 Critical dewand park:

$R_{3}$ between 13 and 15 , Avg expectad domnd: 0.52 Critical operation: $O_{1}^{3}$, Avg. contrib. $100 \%$ $O_{1}^{3}$ scheduled between 14 and 15 on $R_{3}$

>> Dapth: 12, Mnmber of atates risited: 12 Sabedule completed Total Cost: 180

Total Tardines: cost: 55

Total Inventory cont: 125

Avg. Wolghted Tandineas: 2.0

Avg. Welohted Flowtine (WIP) : 10.33

Avg. Melghted Inventory (Flowtine + Earlinens) : 10.42

CPU time: 0.067 veconde

Figure 7, concluded 


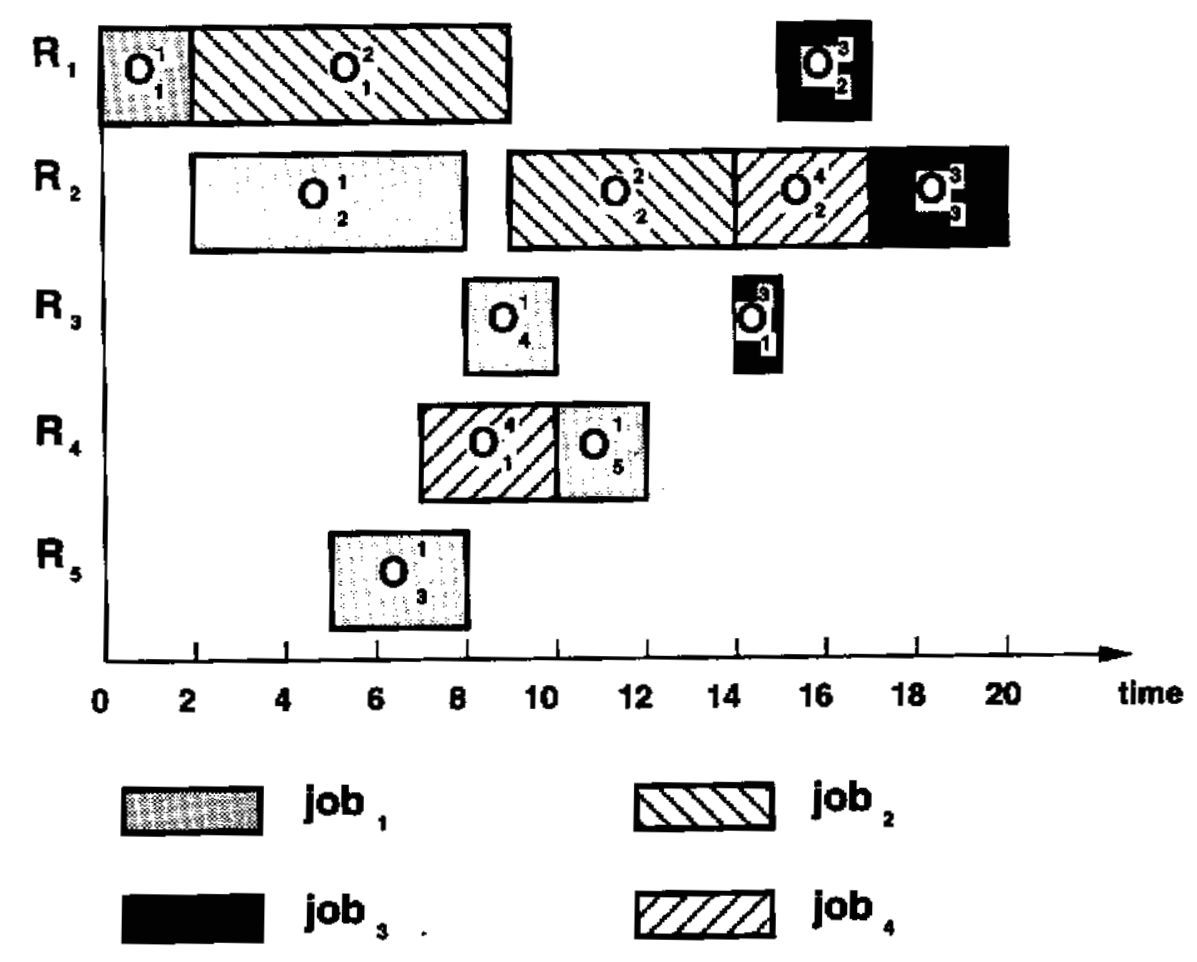

Figure 8: Gantt chart of the final schedule produced by Micro-Boss. 


\section{Reactive and Interactive Scheduling in Micro-Boss}

Manufacturing is a process often fraught with contingencies and subject to a multitude of constraints and preferences that are not always easily amenable to representation in a computer model.

Operation durations tend to vary, machines break down, raw materials fail to arrive on time, new customer orders arrive, others get cancelled, etc. Many ad hoc constraints and preferences that vary over time, such as the preference of a worker on a specific day to perform more demanding tasks in the morning, might be best accounted for via interactive manipulation of the schedule. This section briefly outlines reactive and interactive scheduling capabilities currently under development in the Micro-Boss decision support system.

\subsection{Reactive Scheduling and Control Issues}

Small disruptions, such as minor deviations in operation durations, often do not warrant major modifications to the schedule. However, as the effects of small disruptions accumulate or as more severe disruptions occur, such as long machine breakdowns, it is sometimes desirable to reoptimize the schedule from a more global perspective. Accordingly, in Micro-Boss, schedule disruptions can be handled at two levels based on their severity and the required response time:

1. Control level: Small disruptions that require fast responses are handled by simple control heuristics such as "process the operation with the earliest scheduled start time first" or "when a machine is down, reroute critical jobs to equivalent machines, if any."

2. Scheduling level: In the face of more severe deviations from the schedule, the control level calls upon the Micro-Boss scheduling module to repair/reoptimize the schedule from a more global perspective, while possibly continuing to attend to immediate decisions.

Determining when disruptions should be handed over to the scheduling level can be tricky. Decisions at the control level tend to be rather fast because they are based on local heuristics with a very restricted view of the problem. Decisions at the scheduling level tend to produce better repairs but take longer because they are based on more global considerations. There is generally a trade-off between the responsiveness of the overall system and the amount of reoptimization that can be performed. In manufacturing environments where disruptions are very frequent, a large number of disruptions might need to be handled at the control level, whereas, in less chaotic environments, a larger proportion of disruptions might be processed at the scheduling level. A similar two-tier approach to handling schedule disruptions was first proposed by Smith et al. [36]. Within this approach, the scheduling level restricts the set of alternatives to be considered at the control level by imposing a legal temporal window of execution on each operation. If the controller cannot respect an operation's window of execution, it has to request a new schedule (and a new set of execution windows) from the scheduler. One 
objective of ongoing research in reactive scheduling and control within Micro-Bass is to assess the merits of different coordination regimes between the scheduling and control levels.

Schedule repair in Micro-Boss differs from recent approaches that emphasized the use of iterative repair heuristics $[37,20,44]$. In the process of resolving schedule conflicts, iterative repair heuristics are allowed to introduce new conflicts, which, in turn, require more repairs. This iterative behavior can sometimes lead to myopic decisions and can potentially become expensive. In contrast to these approaches, schedule repair in Micro-Boss attempts to take a more global view of the repair problem and capitalize on the strengths of the micro-opportunistic search procedures in the system. Concretely, schedule repair in Micro-Boss is performed in two phases: (1) a set of operations that need to be rescheduled is identified and all the operations in this set are unscheduled, and (2) the scheduling problem consisting of all these unscheduled operations and the constraints imposed on them by operations that have already been executed or have not been unscheduled is passed to the micro-opportunistic scheduling module described in the previous sections. The set of operations unscheduled in the first phase is selected in such a way that the resulting scheduling problem (i.e., the one solved in phase 2) generally admits a solution. In the event that a feasible schedule could not be built in phase 2, the system needs to return to phase 1 and undo a larger number of operations. In practice, this situation can generally be avoided by unscheduling slightly more operations than apparently required. Although the resulting search space is slightly larger and, hence, might require longer to be explored, it might also contain better repair solutions. Details on conflict propagation heuristics used in MicroBoss to determine which (and how many) operations to reschedule in the presence of contingencies such as machine breakdowns can be found in [32] ${ }^{16}$.

\subsection{Interactive Scheduling with Micro-Boss}

Although the combinatorial complexity of factory scheduling problems is best handled by automatic scheduling procedures such as the ones described earlier in this paper, ad hoc scheduling constraints and preferences that occur very infrequently or change over time are often best accounted for through interactive manipulation of the schedule. Interactive user support should also include mechanisms that help the user identify sources of inefficiency in the schedule (e.g., tardy orders, overloaded resources, etc.) and ways of correcting these inefficiencies (e.g., adding overtime on a set of resources, rerouting some orders, etc.). Through interaction with the system, the user should be able to explore "what-if" scenarios and weigh different alternatives (e.g., decide whether to complete some jobs past their due dates or work overtime).

The Micro-Boss decision support system enables the end-user to interleave both manual and

\footnotetext{
${ }^{16}$ For particularly severe schedule disruptions such as the breakdown of a bottleneck machine over a long time period, we are also considering rescheduling techniques that subdivide the scheduling horizon and only reschedule those operations that are expected to fall within the near future while overlooking conflicts with operations whose execution is expected to take place later.
} 
automatic (micro-opportunistic) scheduling decisions, analyze, edit, save, and compare complete and partial schedules.

Interactive schedule manipulation is performed using an interactive Gantt chart that displays each resource along with the operations to which that resource has been allocated over time (Figure 9). Schedule manipulation is performed under the supervision of the Micro-Boss consistency enforcing module, which enforces consistency with earlier scheduling decisions (manual and automatic). Partial or complete schedules can be saved and compared against each other along different metrics, including total schedule cost, average weighted tardiness, average weighted earliness, Work-In-Process and Work-In-System (which accounts for both Work-InProcess inventory and finished goods inventory). Optimistic estimates are used for partial schedules for which these metrics cannot be computed exactly. By interleaving both manual and automatic scheduling decisions and saving/restoring partial and complete schedules, the user can compare the impact of alternative scheduling decisions and perform "what-if" analyses.

Figure 9 shows a typical view of the Micro-Boss user interface. In this example, the user is getting ready to modify the working schedule displayed in the Gantt chart, by manually unscheduling an operation on which he/she just clicked. Statistics for the working schedule are compared with statistics for the "current" schedule, namely, the schedule currently in force in the system. These statistics are continuously updated as the user edits the schedule. In another window, the user can check information about specific orders (order2 in this example). In yet another window, he/she has elected to rank orders based on their tardiness in the working schedule. Alternative metrics to rank jobs or resources can be selected in the statistics menu (e.g., cost, tardiness, flowtime, resource utilization, etc.). By clicking on boxes displayed in the Gantt chart, the user can directly obtain information on specific operations (e.g., information on operation milling31), manually unschedule and reschedule operations (by moving the corresponding box in the Gantt chart), unschedule jobs, or highlight a job by changing its color. The Gantt menu also allows for zooming in and out of the Gantt chart, unscheduling specific resource/time intervals, displaying contention measures over time for different resources, etc. 


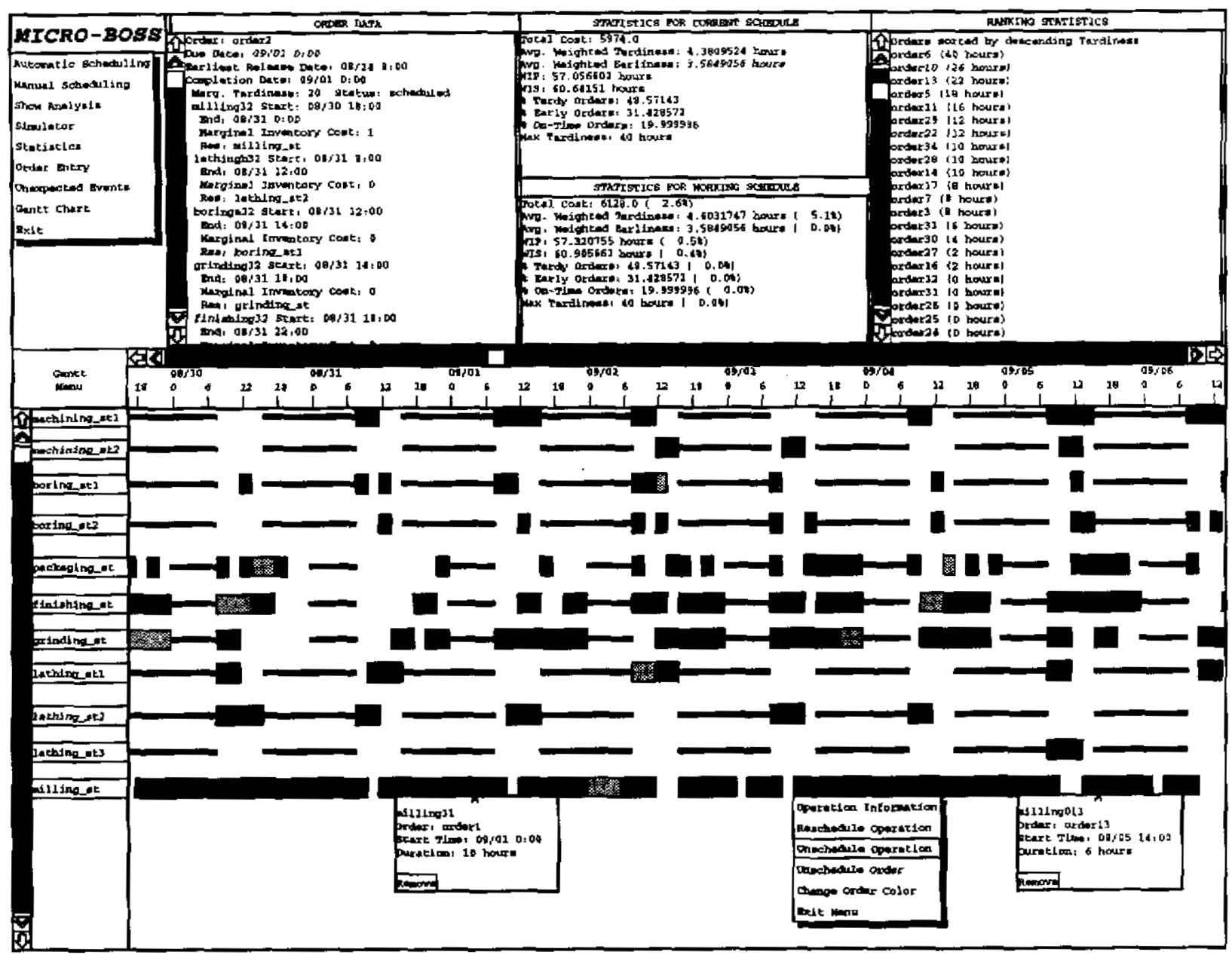

Figure 9: The Micro-Boss user interface allows for interactive manipulation of schedules. By interleaving both manual and automatic scheduling decisions, saving and comparing alternative schedules, the user can easily assess different trade-offs and locally impose ad hoc constraints or preferences that are not easily amenable to representation in the computer model. 


\section{Performance Evaluation}

Experimental studies performed with an initial version of Micro-Boss implemented using Knowledge Craft ${ }^{\mathrm{TM}}$ were reported in [30]. These experiments studied the performance of the system under a variety of scheduling conditions and different cost assumptions ${ }^{17}$. They included comparisons with combinations of popular priority dispatch rules and release policies advocated in the Operations Research literature, comparisons with coarser bottleneck-centered approaches to scheduling described in the Artificial Intelligence literature and a comparison with a variation of Micro-Boss in which resource contention was measured using unbiased demand profiles.

In this paper, we report the results of a similar study performed on the same set of scheduling problems with a more recent version of the system written in $\mathrm{C}++$. At the present time (January 1994), the new version of Micro-Boss is two orders of magnitude faster than the version described in [30] on this set of problems, mainly because of the $\mathrm{C}++$ reimplementation and the use of rough demand profiles to identify small areas of high contention over which more detailed profiles are then constructed (see section 2.4). The new system also uses a more powerful consistency enforcing procedure (See Subsection 2.2) than the original version, which almost eliminates the need for backtracking on the experiments reported in this paper. Finally, the new system also produces schedules that are significantly better than those obtained with the earlier version. This improvement in schedule quality is mainly attributed to the use of a more accurate set of propagation heuristics to update the best remaining start time(s) of unscheduled operations during construction of the schedule and the use of a stronger bias in the construction of demand profiles.

\begin{tabular}{|c|c|c|c|}
\hline \multicolumn{4}{|c|}{ Problem Sets } \\
\hline $\begin{array}{c}\text { Problem } \\
\text { Set }\end{array}$ & $\begin{array}{c}\text { Number of } \\
\text { Bottlenecks }\end{array}$ & $\begin{array}{c}\text { Avg. } \\
\text { Due Date }\end{array}$ & $\begin{array}{c}\text { Due Date } \\
\text { Range }\end{array}$ \\
\hline 1 & 1 & loose & wide \\
\hline $\mathbf{2}$ & 1 & loose & narrow \\
\hline $\mathbf{3}$ & 1 & tight & wide \\
\hline $\mathbf{4}$ & 1 & tight & narrow \\
\hline $\mathbf{5}$ & 2 & loose & wide \\
\hline $\mathbf{6}$ & 2 & loose & narrow \\
\hline $\mathbf{7}$ & 2 & tight & wide \\
\hline $\mathbf{8}$ & 2 & tight & narrow \\
\hline
\end{tabular}

Table 2: Characteristics of the eight problem sets.

The results reported below were obtained on a suite of 80 scheduling problems. The suite,

${ }^{17}$ Knowledge Craft is a registered trademark of Carnegie Group. 
which is described in detail in [30], consists of eight sets of scheduling problems obtained by adjusting three parameters to cover a wide range of scheduling conditions. The three parameters are the following: an average due date parameter (tight versus loose average due date), a due date range parameter (narrow versus wide range of due dates), and a parameter controlling the number of major bottlenecks (in this case one or two). For each parameter combination, a set of 10 scheduling problems was randomly generated (see Table 2), thereby resulting in a total of 80 scheduling problems ( 10 problems $\times 2$ average due date values $\times 2$ due date ranges $\times 2$ bottleneck configurations). Each problem requires scheduling 20 jobs on 5 resources for a total of 100 operations. Marginal tardiness costs in these problems were set to be, on the average, five times larger than marginal inventory costs to model a situation where tardiness costs dominate but inventory costs are non-negligible ${ }^{18}$.

Micro-Boss required between 10 and $15 \mathrm{CPU}$ seconds to schedule each problem on a DECstation $^{\text {TM }} 5000 / 200$. Nearly all problems were solved without any backtracking.

\section{Comparison Against Combinations of Priority Dispatch Rules and Release Policies.}

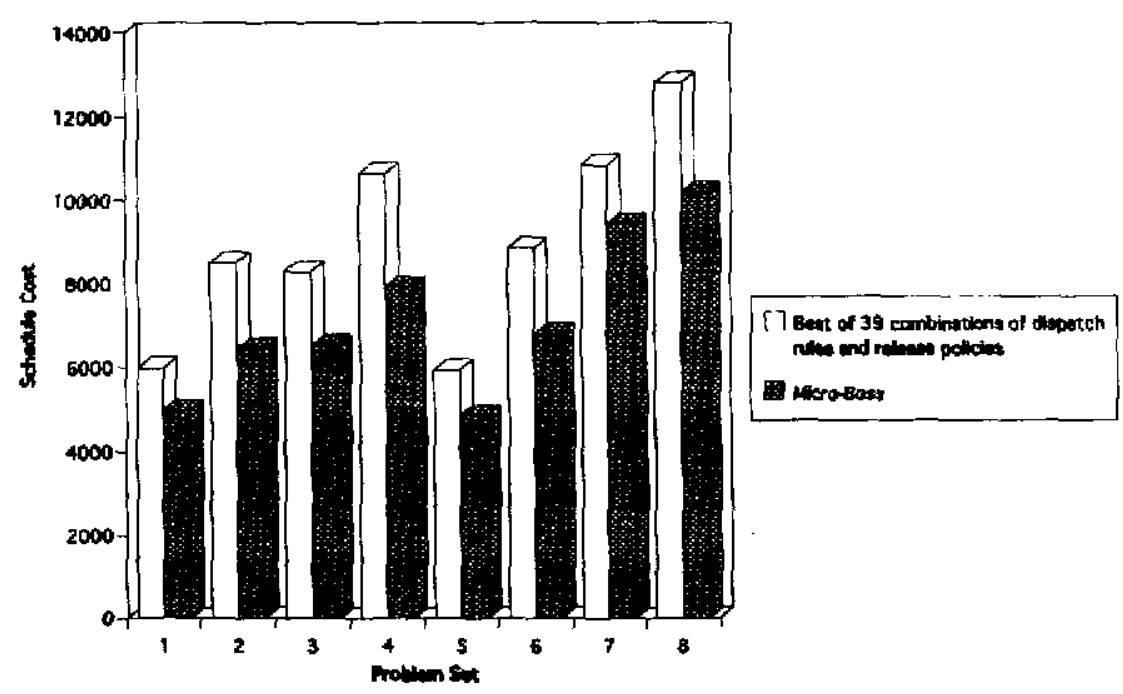

Figure 10: Comparison of Micro-Boss and the best of 39 combinations of priority dispatch rules and release policies under 8 different scheduling conditions ( 10 problems were generated under each condition).

In a first set of experiments, Micro-Boss was compared with the best of a set of 39 combinations of popular priority dispatch rules and release policies. The priority dispatch rules used in these experiments were of two types:

1. a set of five priority dispatch rules that have been reported to be particularly good

\footnotetext{
${ }^{18}$ Experiments under different cost assumptions were also reported in [30].
} 
at reducing tardiness under various scheduling conditions [39]: the Weighted Shortest Processing Time (WSPT) rule; the Earliest Due Date (EDD) rule; the Slack per Remaining Processing Time (S/RPT) rule; and two parametric rules, the Weighted Cost OVER Time (WCOVERT) rule and the Apparent Tardiness Cost (ATC) rule.

2. an exponential version of the parametric early/tardy dispatch rule recently developed by Ow and Morton [26, 21] and referred to below as EXP-ET. This rule differs from the other five in that it can explicitly account for both tardiness and inventory costs.

EXP-ET was successively run in combination with an immediate release policy (IM-REL) that allows each job to be released immediately and with an intrinsic release policy that only releases jobs when their priorities become positive, as suggested in [21]. The other five dispatch rules were successively run in combination with two release policies: an immediate release policy (IM-REL) and the Average Queue Time release policy (AQT) described in [21]. AQT is a parametric release policy that estimates queuing time as a multiple of the average job duration (the look-ahead parameter serving as the multiple). A job's release date is determined by offsetting the job's due date by the sum of its total duration and its estimated queuing time. Combinations of release policies and dispatch rules with a look-ahead parameter were successively run with four different parameter values that generally appeared to produce the best schedules. By combining these different dispatch rules, release policies, and parameter settings a total of 39 heuristics ${ }^{19}$ was obtained. On each problem, the best of the 39 schedules produced by these heuristics was compared with the schedule obtained by Micro-Boss. Among the 39 scheduling heuristics (i.e., excluding Micro-Boss), each of the 6 dispatch rules (WSPT, EDD, S/RPT, WCOVERT, ATC and EXP-ET) and each of the 3 release policies (IM-REL, AQT and EXP-ET's intrinsic release policy) performed best on at least one problem out of the 80 and 12 combinations out of the 39 performed best on at least 1 problem.

Figure 10 compares the average cost of the schedules produced by Micro-Boss with the average cost obtained by the best of the 39 combinations of dispatch rules and release policies on each problem set. Schedule cost was computed as the sum of tardiness and inventory costs, as specified in Equation (3). The results indicate that Micro-Boss consistently outperformed the combination of 39 heuristics under all eight conditions of the study. Overall Micro-Boss yielded reductions of $20 \%$ in schedule cost over the 39 heuristics. A more detailed analysis indicates that this reduction in schedule cost corresponds to a reduction of about $20 \%$ in tardiness costs and

\footnotetext{
${ }^{19}$ The 39 combinations were as follows: EXP-ET and its intrinsic release policy (times four parameter settings), EXP-ET/M-REL (times four parameter settings), EDD/AQT (times four parameter settings), EDD/M-REL, WSPT/AQT (times four parameter settings), WSPT/M-REL, S/RPT/AQT (times four parameter settings), S/RPT/M-REL, WCOVERT/M-REL (times four parameter settings), WCOVERT/AQT (times four parameter settings), ATC/M-REL (times four parameter settings), and ATC/AQT (times four parameter settings).
} 
about $23 \%$ in inventory costs (combined work-in-process and finished goods inventory costs).

\subsection{Comparison Against Coarser Opportunistic Scheduling Procedures}

Micro-Boss was also compared with several coarser opportunistic schedulers that dynamically combine a resource-centered perspective and a job-centered perspective, such as in the OPIS scheduling system [24]. Although OPIS relies on a set of repair heuristics to recover from inconsistencies [25], the macro-opportunistic schedulers of this study were built to use the same consistency enforcing techniques and the same backtracking scheme as Micro-Boss ${ }^{20}$. The macro-opportunistic schedulers also used the same demand profiles as Micro-Boss. When average demand for the most critical resource/time interval was above some threshold level (a parameter of the system that was empirically adjusted), the macro-opportunistic scheduler focused on scheduling the operations requiring that resource/time interval; otherwise, it used a job-centered perspective to identify a critical job and schedule some or all of the operations in that job. Each time a resource/time interval or a portion of a job was scheduled, new demand profiles were computed to decide which scheduling perspective to use next.
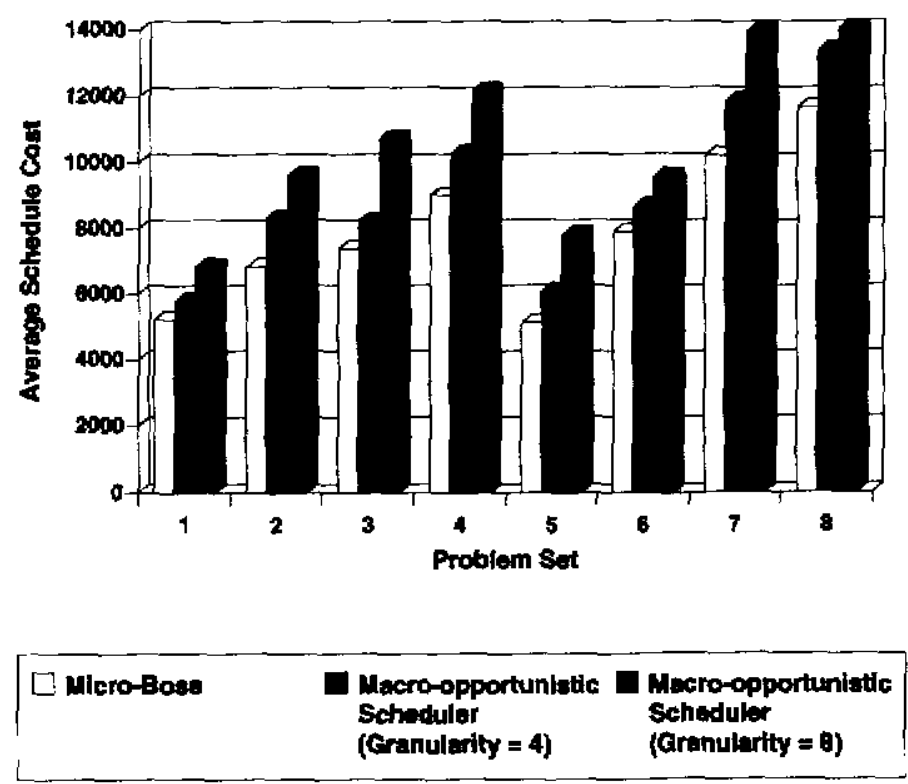

Figure 11: Comparison of Micro-Boss and two coarser opportunistic schedulers.

Figure 11 summarizes the results of a comparison between Micro-Boss ${ }^{21}$ and two macroopportunistic schedulers that differed in the number of operations that they were allowed to

\footnotetext{
${ }^{20}$ An alternative would have been to implement a variation of Micro-Boss using the same repair heuristics as OPIS. Besides being time-consuming to implement, such a comparison would have been affected by the quality of the specific repair heuristics currently implemented in the OPIS scheduler.

${ }^{21}$ These experiments as well as the ones presented in the next subsection were performed in 1993 with an earlier version of Micro-Boss than the one used in the comparison with dispatch rules.
} 
schedule at once in their resource-centered perspective (referred to below as the granularity of the scheduler). The macro-opportunistic scheduler with granularity 4 was allowed to schedule as many as 4 operations in its resource-centered perspective, after which it had to compute new demand profiles and decide which subproblem (job-centered or resource-centered) to focus on next. The macro-opportunistic scheduler with granularity 8 was allowed to schedule at once as many as 8 operations in its resource-centered perspective. The results in Figure 11 indicate not only that Micro-Boss consistently produced better schedules than the two macro-opportunistic schedulers but also that schedule performance degraded as the granularity of the macroopportunistic scheduler was increased, namely, as the search procedure became less flexible. More detailed performance measures not presented here indicate that the reductions in schedule cost achieved by Micro-Boss correspond to reductions in both tardiness and inventory costs.

Overall, these results strongly suggest that the additional flexibility of a micro-opportunistic scheduling procedure over coarser opportunistic procedures generally yields important improvements in schedule quality.

\subsection{Evaluating the Impact of Using Biased Demand Profiles}

A third set of experiments was carried out to test the effect of using biased demand profiles to guide the micro-opportunistic scheduler. A variation of Micro-Boss using unbiased demand profiles was run on the same set of 80 scheduling problems.

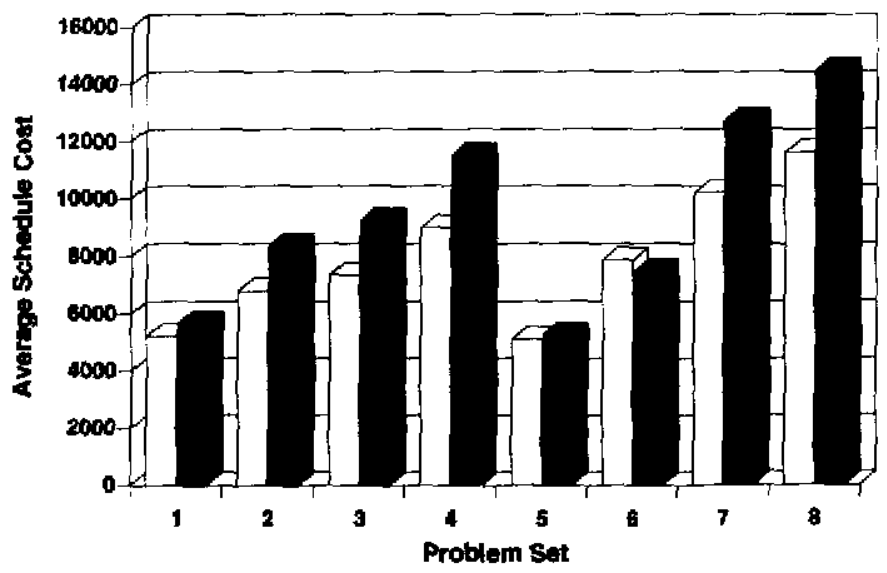

Micro-Boss (biesed version) 1 Unbiased version

Figure 12: Comparison of the cost of the schedules produced by Micro-Boss and a variation of the system that used unbiased demand profiles.

Figure 12 compares the average schedule costs obtained by both variations of Micro-Boss. In

7 out of the 8 scheduling situations of the study, biasing the demand profiles produced reductions 
in schedule cost ranging from 3 to 22 percent, including an impressive 20 percent in the most difficult scheduling situation (Problem Set 8 with two bottlenecks, a tight average due date and a narrow range of due dates). In the one case (out of eight) where the unbiased version produced better schedules, the biased version was only 5\% worse. A more detailed analysis of the results indicates that overall, the biased version of Micro-Boss performed $30 \%$ better with respect to tardiness while incurring a slight increase of $0.6 \%$ in inventory costs. Altogether, biasing the demand profiles reduced schedule costs by more than $15 \%$. These results validate both the idea of building biased demand profiles to guide the micro-opportunistic search procedure and the particular technique used in Micro-Boss to operationalize this idea (namely, the use of the mincost functions). In general, it should be possible to obtain even better results by varying the bias according to specific problem characteristics. One could also consider fine-tuning the bias during the construction of the schedule. 


\section{Concluding Remarks}

Current computer solutions to production management such as the one implemented in MRP/MRP-II systems are of limited help, because they rely on oversimplified models of the plant and only provide weak feedback loops to update the production schedule during execution (typically, complete updates of the schedule are only performed on a weekly basis). A major challenge for researchers in production scheduling is to come up with new techniques that can account more precisely for actual manufacturing objectives and constraints, including execution contingencies such as machine breakdowns, new job arrivals, variations in processing times, yields, etc. New production scheduling tools should also enable the user to interactively perform "what-if" analysis and account for ad hoc constraints and/or preferences that are not easily amenable to representation in the computer model.

In this paper, we presented Micro-Boss, a decision support system for factory scheduling. Micro-Boss aims at combining powerful predictive, reactive, and interactive scheduling capabilities. To this end, the system relies on a new micro-opportunistic search procedure that enables it to continuously track the evolution of micro-bottlenecks (or conflicts) during the construction or repair of the schedule and to refocus its optimization effort on those microbottlenecks that appear most critical. This approach differs from earlier opportunistic approaches $[24,6]$, because it does not require scheduling large resource subproblems or large job subproblems before revising the current scheduling strategy. The results of an experimental study comparing Micro-Boss with combinations of popular priority dispatch rules and release policies advocated in the Operations Research literature as well as coarser opportunistic scheduling approaches proposed in the Artificial Intelligence literature, suggest that the flexibility of this new search procedure can often yield important improvements in schedule quality. We find that because of their flexibility, micro-opportunistic scheduling procedures are also particularly well suited for repairing schedules in the face of execution contingencies and can easily be integrated in interactive decision support systems that enable the user to incrementally manipulate and compare alternative schedules.

Although our work on Micro-Boss has focused on generalized versions of the job shop scheduling problem, micro-opportunistic scheduling techniques have been applied to other manufacturing problems and other classes of problems such as transportation scheduling. Rautaruukki Oy, a large Finnish steel manufacturer, and researchers at the Helsinki University of Technology have reported adapting an earlier version of our micro-opportunistic scheduling heuristics to schedule a steel rolling mill [38]. Variations of the Micro-Boss scheduling heuristics are also used in the Knowledge Based Logistics Planning Shell (KBLPS) developed by Carnegie Group, Inc. (CGI) and LB\&M Associates to solve U.S. army transportation scheduling problems and ammunition distribution planning problems $[8,5,33]$. Other efforts using variations of the micro-opportunistic techniques developed in the context of Micro-Boss are described in $[4,18,28]$ and $[43]$.

Current research efforts within our project aim at applying and extending the existing approach 
to solve both manufacturing and transportation scheduling problems. 


\section{Acknowledgement}

I want to thank Mark Fox for his help and support during the initial development of the system. Special thanks to Bob Schnelbach for his help designing and implementing the MicroBoss user interface and reactive scheduling component and to Shinichi Otsuka for his help with the $\mathrm{C}++$ reimplementation of the system. Recent research efforts have also benefited from the participation of Bryan Lewis, Gang Li, Yoichiro Nakakuki, Katia Sycara, Joe Toomey, Sam Thangiah, and Yalin Xiong. 


\section{References}

[1] J. Adams, E. Balas, and D. Zawack.

The Shifting Bottleneck Procedure for Job Shop Scheduling.

Management Science 34(3):391-401, 1988.

[2] Kenneth R. Baker and Gary D. Scudder.

Sequencing with Earliness and Tardiness Penalties: A Review.

Operations Research 38(1):22-36, January-February, 1990.

[3] Bean, J.C.,J.R. Birge, J. Mittenthal, C.E. Noon.

Matchup Scheduling with Multiple Resources, Release Dates and Disruptions.

Operations Research 39(3):470-483, May-June, 1991.

[4] Pauline M. Berry.

The PCP: A Predictive Model for Satisfying Conflicting Objectives in Scheduling

Problems.

Technical Report, Centre Universitaire d'Informatique, Universite de Geneve, 12, Rue du Lac, CH-1207, Geneva, Switzerland, 1991.

[5] Camden, R., Dunmire, C., Goyal, R., Sathi,N., Elm, B., and Fox, M.

Distribution Planning: An Integration of Constraint Satisfaction and Heuristic Search Techniques.

In Proceedings of the Conference on AI Applications in Military Logistics. 1990.

[6] A. Collinot, C. Le Pape and G. Pinoteau.

SONIA: a Knowledge-based Scheduling System.

International Joumal of Artificial Intelligence in Engineering 2(4):86-94, 1988.

[7] S. Dauzere-Peres and J.B. Lasserre.

A Modified Shifting Bottleneck Procedure for Job Shop Scheduling.

Technical Report LAAS 90106, Laboratoire d'Automatique et d'Analyse des Systemes,

7, Av. du Colonel Roche, 31077 Toulouse Cedex, France, 1990.

[8] Dunmire, C., Sathi,N., Goyal, R., Fox, M., and Kott, A.

Ammunition Inventory Planning: An Integration of Configuration and Resource

Allocation Techniques.

In Proceedings of the Conference on AI Applications in Military Logistics. 1990.

[9] Mark S. Fox.

Constraint-Directed Search: A Case Study of Job-Shop Scheduling.

$\mathrm{PhD}$ thesis, Department of Computer Science, Carnegie-Mellon University, 1983.

[10] R.E. Fox.

OPT: Leapfrogging the Japanese.

Just-in-time Manufacture.

In C.A. Voss,

IFS Ltd, Springer Verlag, 1987.

[11] S. French.

Sequencing and Scheduling: An Introduction to the Mathematics of the Job-Shop. Wiley, 1982. 
[12] M.R. Garey and D.S. Johnson.

Computers and Intractability: A Guide to the Theory of NP-Completeness.

Freeman and Co., 1979.

[13] Eliyahu M. Goldratt.

Optimized Production Timetable: Beyond MRP: Something Better is finally Here.

October, 1980

Speech to APICS National Conference.

[14] Graves, S.C.

A Review of Production Scheduling.

Operations Research 29(4):646-675, July-August, 1981.

[15] F. Robert Jacobs.

OPT Uncovered: Many Production Planning And Scheduling Concepts Can Be Applied With Or Without The Software.

Industrial Engineering 16(10):32-41, October, 1984.

[16] Claude Le Pape and Stephen F. Smith.

Management of Temporal Constraints for Factory Scheduling.

Technical Report, The Robotics Institute, Carnegie Mellon University, Pittsburgh, PA 15213, 1987.

also appeared in Proc. Working Conference on Temporal Aspects in Information Systems, Sponsored by AFCET and IFIP Technical Committee TC8, North Holland Publishers, Paris, France, May 1987.

[17] Gang Li and Norman Sadeh.

Single-Machine Early/Tardy Scheduling Problem with Setups: A Hybrid Heuristic Approach.

Technical Report, Robotics Institute, Carnegie Mellon University, Pittsburgh, PA 15213, 1993.

Working paper. Presented at the Joint National ORSATTIMS meeting held in San Francisco, November 1-4, 1992.

[18] Theodore A. Linden.

Preference-Directed, Cooperative Resource Allocation and Scheduling.

Technical Report, Advanced Decision Systems, 1500 Plymouth St., Mountain View, CA 94043, September, 1991.

[19] A.K. Mackworth and E.C. Freuder.

The Complexity of some Polynomial Network Consistency Algorithms for Constraint Satisfaction Problems.

Artificial Intelligence $25(1): 65-74,1985$.

[20] S. Minton, M.D. Johnston, A.B. Philips, P. Laird.

Solving Large-Scale Constraint Satisfaction and Scheduling Problems Using a Heuristic Repair Method.

In Proceedings of the Eighth National Conference on Artificial Intelligence, pages 17-24. 1990.

[21] T.E. Morton, S.R. Lawrence, S. Rajagopolan, S Kekre.

SCHED-STAR: A Price-Based Shop Scheduling Module.

Journal of Manufacturing and Operations Management :131-181, 1988. 
[22] Joseph Orlicky.

Material Requirements Planning.

McGraw Hill, New York, 1975.

[23] Peng Si Ow.

Focused Scheduling in Proportionate Flowshops.

Management Science 31(7):852-869, 1985.

[24] Peng Si Ow and Stephen F. Smith.

Viewing Scheduling as an Opportunistic Problem-Solving Process.

Annals of Operations Research 12:85-108, 1988.

[25] P.S. Ow, S.F. Smith, and A. Thiriez.

Reactive Plan Revision.

In Proceedings of the Seventh National Conference on Artificial Intelligence, pages

77-82. 1988.

[26] Peng Si Ow and Thomas Morton.

The Single Machine Early/Tardy Problem.

Management Science 35(2):177-191, 1989.

[27] S.S. Panwalkar and Wafik Iskander.

A Survey of Scheduling Rules.

Operations Research 25(1):45-61, January-February, 1977.

[28] Paolucci, E., Patriarca, E., Sem, M., and Gini G.

Predit: A Temporal Predictive Framework for Scheduling Systems.

In Proceedings of the AAAI Spring Symposium on Practical Approaches to Scheduling and Planning, pages 150-154. 1992.

[29] N. Sadeh and M.S. Fox.

Variable and Value Ordering Heuristics for Hard Constraint Satisfaction Problems: an Application to Job Shop Scheduling.

Technical Report CMU-RI-TR-91-23, The Robotics Institute, Carnegie Mellon University, Pittsburgh, PA 15213, 1991.

Submitted to the Artificial Intelligence Journal.

[30] Norman Sadeh.

Look-ahead Techniques for Micro-opportunistic Job Shop Scheduling.

$\mathrm{PhD}$ thesis, School of Computer Science, Carnegie Mellon University, March, 1991.

[31] Norman Sadeh, Katia Sycara, and Yalin Xiong.

Backtracking Techniques for Hard Scheduling Problems.

Technical Report CMU-RI-TR-92-06, The Robotics Institute, Carnegie Mellon University, Pittsburgh, PA 15213, 1992.

Submitted to the Artificial Intelligence Journal.

[32] Sadeh, N.M., S. Otsuka, and R. Schnelbach.

Predictive and Reactive Scheduling with the Micro-Boss Production Scheduling and Control System.

In Proceedings of the IJCAI-93 Workshop on Knowledge-based Production Planning, Scheduling, and Control. Chambery, France, August, 1993. 
[33] Victor Saks, Al Kepner, and Ivan Johnson.

Knowledge Based Distribution Planning.

Technical Report, Carnegie Group, Inc., 5 PPG Place, Pittsburgh, PA 15222, 1992.

[34] P. Serafini, W. Ukovich, H. Kirchner, F. Giardina, and F. Tiozzo.

Job-shop scheduling: a case study.

Operations Research Models in FMS.

In F. Archetti, M. Lucertini, and P. Serafini,

Springer, Vienna, 1988.

[35] S. Smith, M. Fox, and P.S. Ow.

Constructing and Maintaining Detailed Production Plans: Investigations into the

Development of Knowledge-Based Factory Scheduling Systems.

Al Magazine 7(4):45-61, Fall, 1986.

[36] Smith, S.F., N. Keng, and K. Kempf.

Exploiting Local Flexibility During Execution of Pre-Computed Schedules.

Technical Report CMU-TR-RI-90-13, The Robotics Institute, Carnegie Mellon

Univeristy, June, 1990.

[37] Stephen F. Smith, Peng Si Ow, Nicola Muscettola, Jean-Yves Potvin, Dirk Matthys.

An Integrated Framework for Generating and Revising Factory Schedules.

Journal of the Operational Research Society 41(6):539-552, 1990.

[38] Seppo Torma, Ora Lassila and Markku Syrjanen.

Adapting the Activity-Based Scheduling Method to Steel Rolling.

In G. Doumeingts, J. Browne, and M Tomljanovich (editor), Proceedings of the Fourth

IFIP Conference on Computer Applications in Production and Engineering

(CAPE'91), pages 159-166. Elsevier Science Publishers B.V. (North Holland), 1991.

[39] Ari P.J. Vepsalainen and Thomas E. Morton.

Priority Rules for Job Shops with Weighted Tardiness Costs.

Management Science 33(8): 1035-1047, 1987.

[40] Thomas Vollmann, William Berry, and Clay Whybark.

Manufacturing Planning and Control.

Dow Jones-Irwin, Homewood, IL, 1988.

Second Edition.

[41] Oliver Wight.

MRP II: Unlocking America's Productivity Potential.

Oliver Wight Limited Publications, Williston, VT, 1981.

[42] Oliver Wight.

Manufacturing Resource Planning: MRPII.

Oliver Wight Limited Publications, Essex Junction, VT, 1984.

[43] Andreas Winklhofer, Manfred Maierhofer, and Paul Levi.

Efficient Propagation and Computation of Problem Features for Activity-Based Scheduling.

In Proceedings of the Seventh Symposium on Information Control Problems in Manufacturing Technology (INCOM-92). Toronto, Canada, 1992. 
[44] Monte Zweben, Eugene Davis, and Michael Deale.

Iterative Repair for Scheduling and Rescheduling.

Technical Report, NASA Ames Reserch Center, MS 244-17, Moffett Field, CA 94035, 1991. 
44 $|d|$ 4 